Page 1 of 4 Proj.

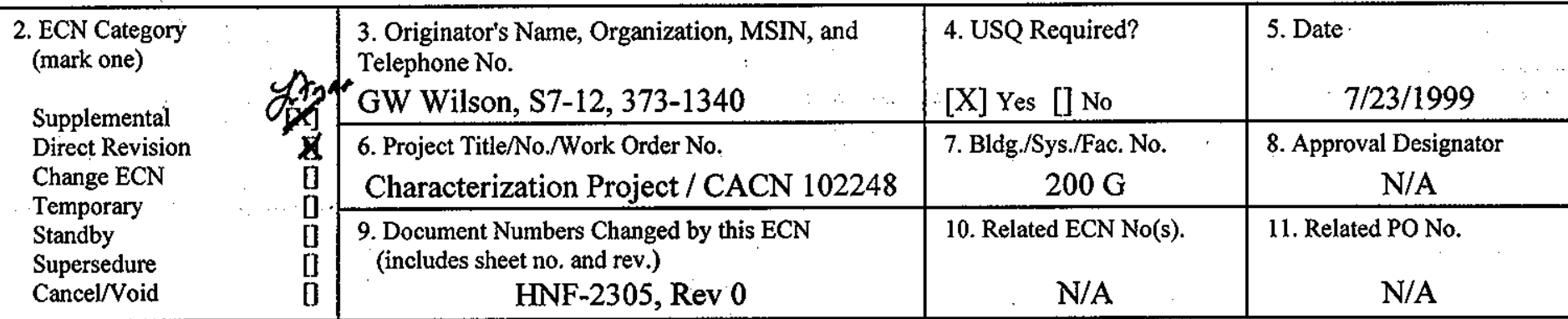

\begin{tabular}{|c|c|c|c|}
\hline 12a: Modification Work & $\begin{array}{l}\text { 12b. Work Package } \\
\text { No. }\end{array}$ & 12c. Modification Work Complete & $\begin{array}{l}\text { 12d. Restored to Original Condition (Temp. } \\
\text { or Standby ECN only) }\end{array}$ \\
\hline [] Yes (fill out Blk. 12b) & N/A & N/A & N/A \\
\hline $\begin{array}{c}{[\mathrm{X}] \text { No (NA Blks. 12b, }} \\
12 \mathrm{c}, 12 \mathrm{~d})\end{array}$ & & $\begin{array}{l}\text { Design Authority/Cog. Engineer } \\
\text { Signature \& Date }\end{array}$ & $\begin{array}{l}\text { Design Authority/Cog. Engineer } \\
\text { Signature \& Date }\end{array}$ \\
\hline
\end{tabular}

13a. Description of Change

13b. Design Baseline Document? [] Yes [X] No

ETN-98-0001

\title{
DRAWING EVALUATION REPORT FOR SAMPLING EQUIPMENT
}

This ECN updates the drawing evaluation report to reflect drawing status changes and procedure changes.

See attached pages for details.

14a. Justification (mark one)

Criteria Change [X]

As-Found

[]

Design Improvement []

Environmental

[]

Const. Error/Omission []

Facility Deactivation

Design Error/Omission []

14b. Justification Details

* A yearly update of the evaluation report is required to ensure procedure compliance.

* This ECN is covered by USQ TF-96-690, Rev. 2

* Design verification performed by informal review per HNF-IP-0842, VOL IV, SEC. 4.24

* This modification will not change collective dose since it has no impact on radiological sources; contamination control, or shielding.

* The changes implemented by this ECN do not modify any sampling equipment. Therefore, a NEPA screening is not required per section 2.1 of HNF-PRO-452.

\begin{tabular}{|c|c|c|c|c|c|}
\hline \multicolumn{6}{|c|}{ 15. Distribution (include name, MSN, and no. of copies) } \\
\hline M.E. Beaver & S7-12 & $1 \mathrm{COPY}$ & E. E. Șalinas & S7-12 & $1 \mathrm{COPY}$ \\
\hline R. M. Boger & S7-12 & $1 \mathrm{COPY}$ & J. L. Smalley. & S7-12 & $1 \mathrm{COPY}$ \\
\hline R. N. Dale & S7-12 & $1 \mathrm{COPY}$ & G. W. Wilson & S7-12 & $1 \mathrm{COPY}$ \\
\hline G. P. Janicek & $\$ 7-12$ & $1 \mathrm{COPY}$ & & & \\
\hline
\end{tabular}


ENGINEERING CHANGE NOTICE

1. ECN (use no. from pg. l)

\begin{tabular}{|c|c|c|c|c|c|c|c|c|}
\hline $\begin{array}{l}\text { 16. Design } \\
\text { Verification } \\
\text { Required }\end{array}$ & \multicolumn{3}{|c|}{$\begin{array}{l}\text { 17. Cost Impact } \\
\text { ENGINEERING }\end{array}$} & \multicolumn{3}{|c|}{ CONSTRUCTION } & \multicolumn{2}{|c|}{ 18. Schedule Impact (days) } \\
\hline$[\mathrm{X}]$ Yes & Additional & [N/A] & $\$$ & Additional & {$[\mathrm{N} / \mathrm{A}]$} & $\$$ & Improvement & [N/A] \\
\hline [] No & Savings & [] & $\$$ & Savings & [] & $\$$ & Delay & [] \\
\hline
\end{tabular}

651956

19. Change Impact Review: Indicate the related documents (other than the engineering documents identified on Side 1) that will be affected by the change described in Block 13. Enter the affected document number in Block 20.

$\begin{array}{lllll}\text { SDD/DD } & {[]} & \text { Seismic/Stress Analysis } & {[]} & \text { Tank Calibration Manual } \\ \text { Functional Design Criteria } & {[]} & \text { Stres/Design Report } & {[]} & \text { Health Physics Procedure } \\ \text { Operating Specification } & {[[]} & \text { Interface Control Drawing } & {[]} & \text { Spares Multiple Unit Listing } \\ \text { Criticality Specification } & {[]} & \text { Calibration Procedure } & {[]} & \text { Test Procedures/Specification } \\ \text { Conceptual Design Report } & {[]} & \text { Installation Procedure } & {[]} & \text { Component Index } \\ \text { Equipment Spec. } & {[]} & \text { Maintenance Procedure } & {[]} & \text { ASME Coded Item } \\ \text { Const. Spec. } & {[]} & \text { Engineering Procedure } & {[]} & \text { Human Factor Consideration } \\ \text { Procurement Spec. } & {[]} & \text { Operating Instruction } & {[]} & \text { Computer Software } \\ \text { Vendor Information } & {[]} & \text { Operating Procedure } & {[]} & \text { Electric Circuit Schedule } \\ \text { OM Manual } & {[]} & \text { Operational Safety Requirement } & {[]} & \text { ICRS Procedure } \\ \text { FSAR/SAR } & {[]} & \text { IEFD Drawing } & {[]} & \text { Process Control Manual/Plan } \\ \text { Safety Equjpment List } & {[} & \text { Cell Arrangement Drawing } & {[]} & \text { Process Flow Chart } \\ \text { Radiation Work Permit } & {[]} & \text { Essential Material Specification } & {[]} & \text { Purchase Requisition } \\ \text { Environmental Impact Statement } & {[]} & \text { Fac. Proc. Samp. Schedule } & {[]} & \text { Tickler File } \\ \text { Environmental Report } & {[]} & \text { Inspection Plan } & {[]} & \text { None } \\ \text { Environmental Permit } & {[]} & \text { Inventory Adjustment Request } & {[]} & \end{array}$

[]
[]
[]
[]
[]
[]
[]
[]
[]
[]
[]
[]
[]
[]
[]
$[\mathrm{X}]$

20. Other Affected Documents: (NOTE: Documents listed below will not be revised by this ECN.) Signatures below indicate that the signing organization has been notified of other affected documents listed below.

Document Number/Revision

N/A
Document Number/Revision

N/A
Document Number Revision N/A

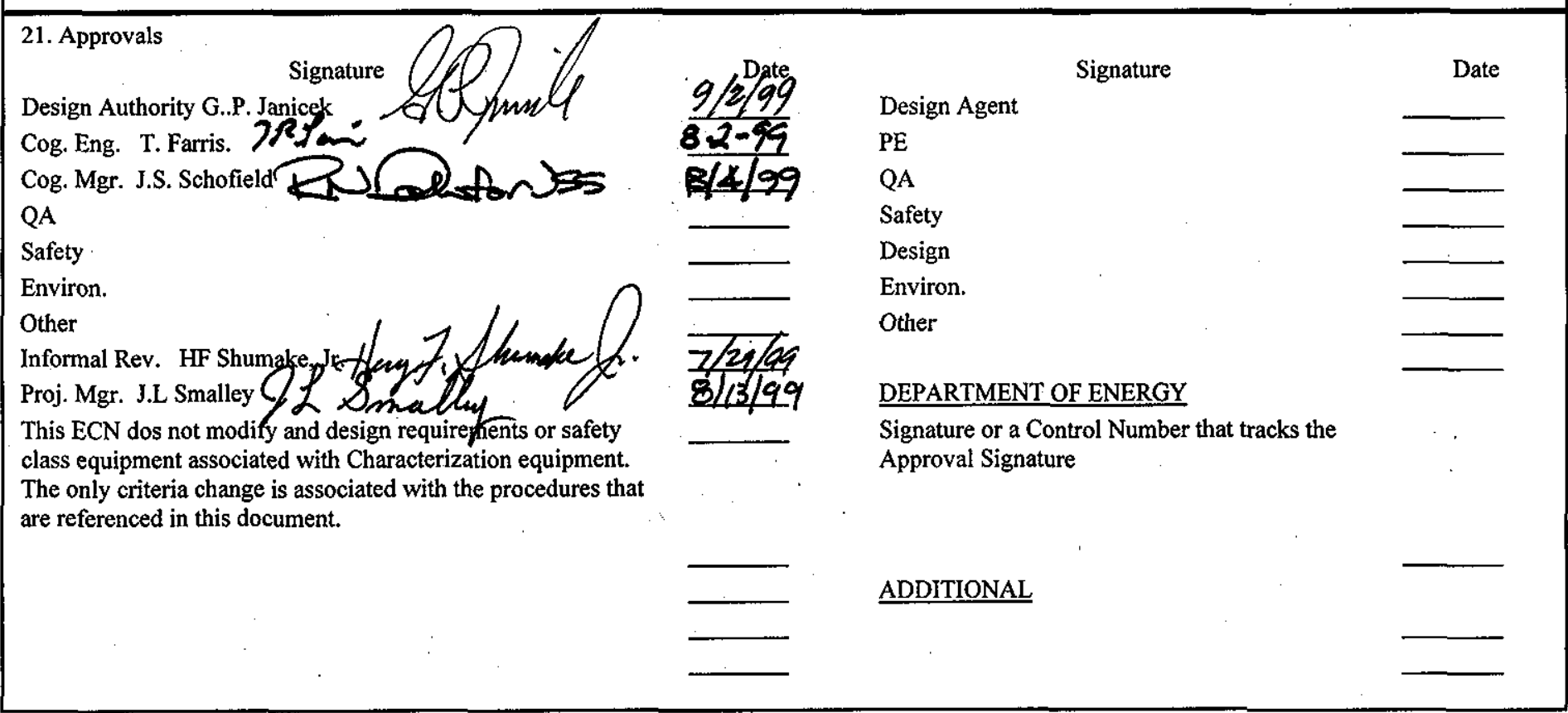




\section{ENGINEERING CHANGE NOTICE CONTINUATION SHEET}

HNF-2305: Change the document as outlined below;

\section{Section 1.0}

- ....Revised paragraph to reflect that this is an update to the evaluation.

- Revised paragraph to change from TWRS to River Protection Project

Section 2.0

- Revised to indicate that this is an update of the drawings evaluation

- Added Light Duty Utility Arm (LDUA) drawings

- Changed old task order to current contract number

\section{Section 3.0}

- Revised reference for Engineering Drawing to current procedure

- Revised to change from TWRS to RPP

Section 4.0

- Added section for LDUA

Section 4.1

- Corrected category for $\mathrm{H}-2-38075$ from $\mathrm{S}$ to $\mathrm{G}$

- Deleted drawings H-2-90257, H-2-91722, H-2-91723, H-2-91724, and H-2-91725 which are outdated drawings that were cancelled.

- Changed summary, sec. 4.1.1., to reflect noted changes

Section 4.2

- Updated number of sheets for $\mathrm{H}-2-91707$ from 2 to 3

- Updated summary, sec. 4.2.1., to reflect noted changes

\section{Section 4.3}

- Changed all drawings that previously were categorized as S or E to category $\mathrm{G}$

- Updated number of sheets for H-2-89462 from 1 to 3

- Updated summary, sec. 4.3.1., to reflect noted changes

Section 4.4

- Corrected number of sheets for H-2-827188 from 7 to 9, H-2-827192 from 1 to 2 , and H-2-827193 from 1 to 2

- Updated summary, sec. 4.4.1., to reflect noted changes

Section 4.5

- Added drawings H-2-75053, H-2-85349, H-2-85349, $\mathrm{H}-2-99346, \mathrm{H}-2-690126$, and $\mathrm{H}-2-690144$.

- Updated number of sheets for H-2-38078 from 1 to 2, H-2-85299 from 1 to 7, H-2-85341 from 1 to 2 , and $\mathrm{H}-2-690124$ from 2 to 4. 


\section{ENGINEERING CHANGE NOTICE CONTINUATION SHEET}

- Changed category for $\mathrm{H}-9-174$ from $\mathrm{E}$ to $\mathrm{G}$

- Added H-2-85299, sheets $8,9, \& 10$ as category $\mathrm{E}$

- Deleted drawings H-2-91679, H-2-91680, and H-2-99316

- Updated Summary, sec. 4.5.1., to reflect noted changes

Section 4.6

- Deleted drawings H-14-0030122, H-14-100739, H-14-100741, H-2-821455, and H-2-821456

- Updated number of drawing sheet for H-2-829082 from 2 to $6, \mathrm{H}-829108$ from 4 to 5, H-2-829121 from 2 to 6 , and $\mathrm{H}-2-827137$ from 4 to 5

- Added drawing H-2-829109 and H-2-829138

- Updated Summary, sec. 4.6.1., to reflect noted changes

Section 4.7

- Changed category for all auger sampling drawings (H-2-79960 \& H-2-826321) from $S$ to $G$

- Updated Summary, sec. 4.7.1., to reflect noted changes

Section 4.8

- . Added entire section and summary for LDUA drawings ( see document for details)

Section 5.0

- Updated table 5.1 to reflect changes in total number of drawings

- Revised summary to note that Truck $3 \& 4$, exhauster, and support drawings comply with drawing categories noted in this document and that Truck 1 does not comply. 


\title{
DRAWING EVALUATION REPORT FOR SAMPLING EQUIPMENT DRAWINGS
}

\author{
R. M. Boger \\ Lockheed Martin Hanford Corporation \\ Richland, WA 99352 \\ U.S. Department of Energy Contract DE-AC06-96RL13200
}

EDT/ECN: 651956

Org Code: 74900

B\&R Code: EW3120074
UC: 2070

Charge Code: 102248/BO00

Total Pages: 46

Key Words: Sampling, Drawing Evaluation, Category, RMCST

Abstract: This report documents the reuslts of an evaluation of Characterization sampling equipment drawigns for compliance with existing procedures on establishing drawing categories.

TRADEMARK DISCLAIMER. Reference herein to any specific commercial product, process, or service by trade name, trademark, manufacturer, or otherwise, does not necessarily constitute or imply its endorsement, recommendation, or favoring by the United States Government or any agency thereof or its contractors or subcontractors.

ichland WA 99352, Phone (509) 372-2420; Fax (509) 376-4989.
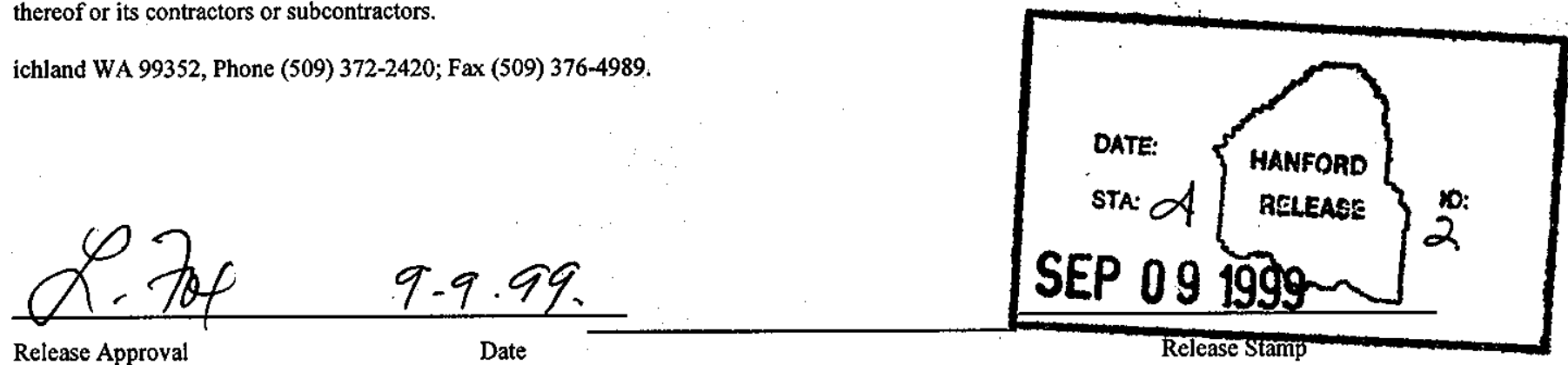

\section{Approved for Public Release}




\begin{tabular}{|c|c|c|c|}
\hline \multicolumn{2}{|r|}{ RECORD OF REVISION } & \multicolumn{2}{|c|}{$\begin{array}{r}\text { (1) Document Number } \\
\text { HNF-2305 }\end{array}$} \\
\hline \multicolumn{4}{|c|}{$\begin{array}{l}\text { (2) Title } \\
\text { Drawing Evaluation Report For Sampling Equipment }\end{array}$} \\
\hline \multicolumn{4}{|c|}{ CHANGE CONTROL RECORD } \\
\hline \multirow{3}{*}{ (3) Revision } & \multirow{2}{*}{ (4) Description of Change - Replace, Add, and Delete Pages } & \multicolumn{2}{|c|}{ Authorized for Release } \\
\hline & & (5) Cog. Engr. & \begin{tabular}{|l} 
(6) Cog. Mgr. \\
\end{tabular} \\
\hline & $\begin{array}{l}\text { (7) HNF-2305 "Drawing Evaluation Report For Sampling } \\
\text { Equipment", EDT-623329, 09/15/98 }\end{array}$ & $\begin{array}{l}\text { R.N. Dale } \\
\text { 09/15/98 }\end{array}$ & J.S. Schofield $09 / 15 / 98$ \\
\hline RS & $\begin{array}{l}\text { Incorporate ECN-651956 to perform direct revision for yearly } \\
\text { update. }\end{array}$ & T. R.Farris & J.S. Schafield $9 \%$ \\
\hline & & & \\
\hline & & & \\
\hline & & & \\
\hline & & & \\
\hline & & & \\
\hline & & & \\
\hline & & & \\
\hline & & & \\
\hline & & & \\
\hline & & & \\
\hline & & & \\
\hline & & & \\
\hline & & & \\
\hline & & & \\
\hline & & & \\
\hline & & & \\
\hline & & & \\
\hline & & & \\
\hline & & & - \\
\hline & & & \\
\hline & & & \\
\hline & & & \\
\hline
\end{tabular}


HNF-2305

Rev. 1

\section{DRAWING EVALUATION REPORT}

FOR SAMPLING EQUIPMENT

Prepared For

River Protection Project

Characterization Project

Lockheed Martin Hanford Corporation

Prepared by

G. W. Wilson

COGEMA ENGINEERING CORPORATION

June 1999 
HNF-2305

Rev. 1

CONTENTS

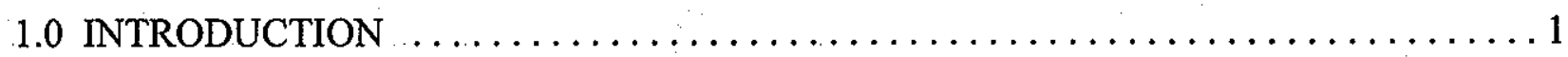

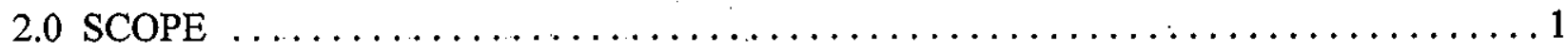

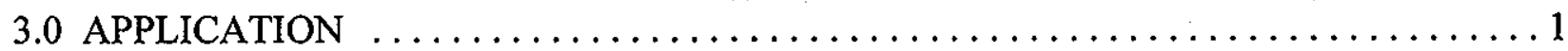

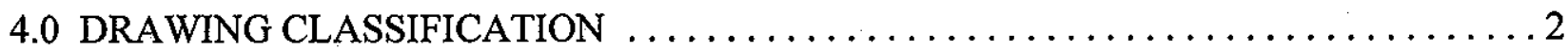

4.1 CORE SAMPLE TRUCK 1 DRAWINGS $\ldots \ldots \ldots \ldots \ldots \ldots \ldots \ldots \ldots$

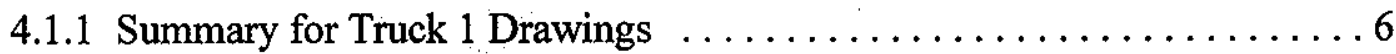

4.2 CORE SAMPLE TRUCK 1 and 2 DRAWINGS $\ldots \ldots \ldots \ldots \ldots \ldots \ldots, 7$

4.2.1 Summary for Truck $1 \& 2$ Drawings $\ldots \ldots \ldots \ldots \ldots \ldots \ldots \ldots$

4.3 CORE SAMPLE TRUCK 2 DRAWINGS $\ldots \ldots \ldots \ldots \ldots \ldots$

4.3.1 Summary of Core Sample Truck $2 \ldots \ldots \ldots \ldots \ldots \ldots \ldots \ldots \ldots \ldots$

4.4 CORE SAMPLE TRUCK $3 \& 4$ DRAWINGS $\ldots \ldots \ldots \ldots \ldots \ldots \ldots \ldots$

4.4.1 Summary RMCST $3 \& 4$ Drawings ...................... 18

4.5 CORE SAMPLING SUPPORT EQUIPMENT DRAWINGS $\ldots \ldots \ldots \ldots \ldots$

4.5.1 Summary for Core Sampling Support Equipment Drawings $\ldots \ldots \ldots 26$

4.6 RMCST SAMPLING EXHAUSTER DRAWINGS $\ldots \ldots \ldots \ldots \ldots \ldots \ldots 27$

4.6.1 Summary for RMCST Exhauster Drawings $\ldots \ldots \ldots \ldots \ldots \ldots \ldots$

4.7 OTHER SAMPLING EQUIPMENT DRAWINGS $\ldots \ldots \ldots \ldots \ldots \ldots \ldots$

4.7.1 Summary for Other Sampling Equipment Drawings $\ldots \ldots \ldots \ldots \ldots . \ldots 32$

4.8 LIGHT DUTY UTILITY ARM (LDUA) DRAWINGS $\ldots \ldots \ldots \ldots \ldots \ldots 33$

4.8.1 Summary for Light Duty Utility Arm Drawings $\ldots \ldots \ldots \ldots \ldots \ldots 40$

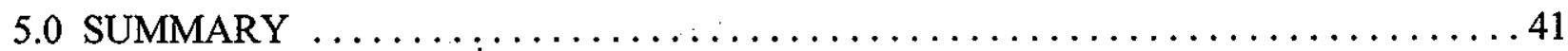

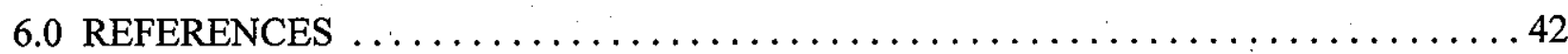


HNF-2305

Rev. 1

\subsection{INTRODUCTION}

This document presents the results of a task to update the evaluation of River Protection Project (RPP) sampling equipment drawings and updates the assigned drawings category as either essential, support, or general drawings. This report updates the drawing evaluation that was originally done per Engineering Task Plan For Truck 3 \& 4 Drawing Compliance and Evaluation (Wilson, 1997).

\subsection{SCOPE}

The scope of this report is limited to updating the evaluation and identification of drawing category for drawings of certain tank waste sampling equipment for which the RPP Characterization Project has been assigned custody, including: vapor sampling, grab sampling, auger sampling, all core sampling equipment, and Light Duty Utility Arm (LDUA) (see LMHC contract \#519, release 10). This report does not address drawings for other waste tank deployed equipment systems having similar assigned custody, such as, Cone Penetrometer system, or Long Length Contaminated Equipment (LLCE). The Cone Penetrometer system, which is depicted on vendor drawings; (not $\mathrm{H}$ - series), is not currently turned over to operations for deployment. The LLCE equipment was just recently assigned to Characterization Project and was not included in the original scope for this update and will be addressed in the evaluation update scheduled for later in fiscal year 1999, when equipment ownership is determined.

\subsection{APPLICATION}

Engineering Drawings (HNF 1999a) defines the drawing categories as follows.

a. Essential - Drawings necessary to directly support the safe operation or maintenance of the facility.

b. Support - Drawings that further describe the design details of structures, systems, or components shown on essential drawings or are frequently used by the support staff. 
HNF-2305

Rev. 1

c. .... General - Drawings that are historical in nature or not frequently used in the operation or maintenance of the facility.

All RPP sampling equipment is mobile equipment that is not directly associated with any one facility. Therefore, this application implemented the requirements of Engineering Drawings (HNF 1999a) tempered by the fact that this equipment is not a permanent on-line facility.

The drawings that are identified as essential were selected by the following criteria:

*. Drawings that describe equipment required for safe operations (drill string arrangements)

* Drawings that depict flow diagrams (hydraulic, pneumatic, and P\&ID diagrams)

* Drawings that depict electrical connections (elementary and one-line diagrams)

* Drawings that depict electrical power connection (one-line and connection diagrams)

\subsection{DRAWING CLASSIFICATION}

The following sections are a detailed listing of the drawings that were evaluated and assigned drawing category. The listings shows the drawing category assigned in the "Cat." column with an $E$ for drawings that are determined to be essential drawings, an $S$ for support drawing, and a $G$ for general drawings. For specific information about equipment drawings, refer to the specified section of this document.

Core Sample Truck 1 Drawings ......................... See Section 4.1

Core Sample Truck 1 and 2 Drawings ........................ See Section 4.2

Core Sample Truck 2 Drawings . . . . . . . . . . . . . . . . . . . . . . See Section 4.3

RMCST Truck 3 and 4 Drawings $\ldots \ldots \ldots \ldots \ldots \ldots \ldots \ldots \ldots$ See Section 4.4

Core Sampling Support Equipment Drawings $\ldots \ldots \ldots \ldots \ldots \ldots \ldots$ See Section 4.5

RMCST Exhauster Drawings $\ldots \ldots \ldots \ldots \ldots \ldots \ldots \ldots \ldots \ldots \ldots \ldots \ldots \ldots \ldots$ See Section 4.6

Other Sampling Equipment Drawings $\ldots \ldots \ldots \ldots \ldots \ldots \ldots \ldots \ldots \ldots$ See Section 4.7

Light Duty Utility Arm $\ldots \ldots \ldots \ldots \ldots \ldots \ldots \ldots \ldots \ldots \ldots \ldots \ldots \ldots \ldots \ldots$ See Section 4.8 
HNF-2305

Rev. 1

\subsection{CORE SAMPLE TRUCK 1 DRAWINGS}

Table 4.1 Core Sample Truck 1 Drawings

\begin{tabular}{|c|c|c|c|c|}
\hline Dwg. No. & Sht. & Description & Cat: & Justification/Notes \\
\hline H-2-38075 & $1-5$ & Shielded Receiver Assembly & $\mathrm{G}$ & Not installed or used \\
\hline $\mathrm{H}-2-81790$ & 1 & $\begin{array}{l}\text { Hydrostatic Head Addition } \\
\text { System Flow Diagram }\end{array}$ & $\mathrm{G}$ & Installed, not used \\
\hline H-2-81832 & $1-2$ & $\begin{array}{l}\text { Drill Rig Junction Box Enclosure } \\
\text { Assembly }\end{array}$ & G & \\
\hline $\mathrm{H}-2-81833$ & $1-2$ & $\begin{array}{l}\text { Hydraulic Bottom Detector Alarm } \\
\text { Panel Assembly }\end{array}$ & $\mathrm{S}$ & \\
\hline $\mathrm{H}-2-91390$ & $1-2$ & Grapple Hoist Installation & $\mathrm{S}$ & $\begin{array}{l}\text { Note: Important for } \\
\text { Maintenance }\end{array}$ \\
\hline H-2-91391 & $1-5$ & Grapple Hoist Assembly & $S$ & $\begin{array}{l}\text { Note: Important for } \\
\text { Maintenance }\end{array}$ \\
\hline $\mathrm{H}-2-91392$ & $1-5$ & Grapple Hoist Box Assembly & S & $\begin{array}{l}\text { Note: Important for } \\
\text { Maintenance }\end{array}$ \\
\hline $\mathrm{H}-2-91393$ & 1 & $\begin{array}{l}\text { Grapple Hoist Box Housing } \\
\text { Assembly }\end{array}$ & G & \\
\hline $\mathrm{H}-2-91394$ & 1 & Encoder Panel Assembly & G & \\
\hline $\mathrm{H}-2-91395$ & 1 & Core Sampler Truck Details & G & Not Used \\
\hline $\mathrm{H}-2-91397$ & $1-4$ & Pressure Readout Display Cabinet & G & \\
\hline $\mathrm{H}-2-91477$ & 1 & Shielded Receiver Assembly & G & Not Used \\
\hline $\mathrm{H}-2-91583$ & 1 & Quill Rod Stop Truck 1 & G & \\
\hline $\mathrm{H}-2-91650$ & 1. & $\begin{array}{l}\text { Core Sampler Truck } 1 \text { Drawing } \\
\text { Index }\end{array}$ & $\mathrm{S}$ & \\
\hline $\mathrm{H}-2-91651$ & $1-3$ & Shielded Receiver Assembly & G & \\
\hline $\mathrm{H}-2-91652$ & 1 & $\begin{array}{l}\text { Shielded Receiver Weather Cover } \\
\text { Assembly }\end{array}$ & G & \\
\hline
\end{tabular}


HNF-2305

Rev. 1

Table 4.1 Core Sample Truck 1 Drawings

\begin{tabular}{|c|c|c|c|c|}
\hline Dwg. No. & Sht. & Description & Cat. & Justification/Notes \\
\hline H-2-91653 & $1-3$ & Sampler Hoist Assembly & $\mathrm{S}$ & $\begin{array}{l}\text { Note: Important for } \\
\text { Maintenance }\end{array}$ \\
\hline H-2-91654 & $1-2$ & Sampler Hoist Housing Assembly & $\mathrm{G}$ & \\
\hline H-2-91655 & $1-2$ & $\begin{array}{l}\text { Sampler Hoist Load Stand } \\
\text { Assembly }\end{array}$ & G & \\
\hline H-2-91656 & 1 & $\begin{array}{l}\text { Sampler Hoist Cable Wiper } \\
\text { Assembly }\end{array}$ & G & \\
\hline $\mathrm{H}-2-91657$ & 1 & $\begin{array}{l}\text { Sampler Hoist Cable Drum. } \\
\text { Assembly }\end{array}$ & G & \\
\hline H-2-91658 & 1 & $\begin{array}{l}\text { Sampler Hoist Cable Feed } \\
\text { Assembly }\end{array}$ & G & \\
\hline H-2-91659 & 1 & Sampler Hoist Cover Assembly & G & \\
\hline H-2-91660 & 1 & $\begin{array}{l}\text { Shielded Receiver Mount } \\
\text { Assembly }\end{array}$ & G & \\
\hline H-2-91661 & 1 & $\begin{array}{l}\text { Sampler Hoist Cable Guide } \\
\text { Assembly }\end{array}$ & G & . \\
\hline H-2-91663 & $1-3$ & Shielded Receiver Base Assembly & G & \\
\hline $\mathrm{H}-2-91664$ & 1 & $\begin{array}{l}\text { Shielded Receiver Tube Section } \\
\text { Assembly }\end{array}$ & $\mathrm{G}$ & \\
\hline H-2-91686 & $1-2$ & $\begin{array}{l}\text { Grapple Hoist Rotary Valve } \\
\text { Actuator }\end{array}$ & $\mathrm{S}$ & $\begin{array}{l}\text { Note: Important for } \\
\text { Maintenance }\end{array}$ \\
\hline $\mathrm{H}-2-91688$ & 1 & Quill Rod Adapter Truck 1 & G & Not used \\
\hline $\mathrm{H}-2-91701$ & $1-7$ & $\begin{array}{l}\text { Truck Assembly Core Sampler } \\
\text { Truck \#1 }\end{array}$ & $S$ & $\begin{array}{l}\text { Note: Important for } \\
\text { Maintenance }\end{array}$ \\
\hline $\mathrm{H}-2-91701$ & 8 & $\begin{array}{l}\text { Truck Assembly Core Sampler } \\
\text { Truck \#1 }\end{array}$ & $\mathrm{E}$ & $\begin{array}{l}\text {-Depicts Equipment } \\
\text { Essential to Safe Operation } \\
\text { (Z-Purge Diagram) }\end{array}$ \\
\hline
\end{tabular}


HNF-2305

Rev. 1

Table 4.1 Core Sample Truck 1 Drawings

\begin{tabular}{|c|c|c|c|c|}
\hline Dwg. No. & Sht. & Description & Cat. & Justification/Notes \\
\hline $\mathrm{H}-2-91701$ & $9-10$ & $\begin{array}{l}\text { Truck Assembly Core Sampler } \\
\text { Truck \#1 }\end{array}$ & $\mathrm{S}$ & $\begin{array}{l}\text { Note: Important for } \\
\text { Maintenance }\end{array}$ \\
\hline H-2-91714 & $1-2$ & $\begin{array}{l}\text { Drill Head Service Platform \#1 } \\
\text { Assembly }\end{array}$ & G & \\
\hline $\mathrm{H}-2-91719$ & $1-3$ & Hydraulic Installation & $\mathrm{S}$. & $\begin{array}{l}\text { Note: Important for } \\
\text { Maintenance }\end{array}$ \\
\hline H-2-91720 & $1-3$ & $\begin{array}{l}\text { Core Sampler Truck \#1 Hydraulic } \\
\text { Flow Diagram }\end{array}$ & E & $\begin{array}{l}\text {-Depicts High-Pressure } \\
\text { Process Diagram }\end{array}$ \\
\hline H-2-91721 & $1-3$ & Drillhead Hydraulic Piping Install & $\mathrm{S}$ & $\begin{array}{l}\text { Note: Important for } \\
\text { Maintenance }\end{array}$ \\
\hline $\mathrm{H}-2-92264$ & 1 & $\begin{array}{l}\text { Control and Power Cable } \\
\text { Assembly }\end{array}$ & G & \\
\hline $\mathrm{H}-2-92315$ & 1 & $\begin{array}{l}\text { Hydraulic and Sampler Actuator } \\
\text { Control Panel }\end{array}$ & G & \\
\hline $\mathrm{H}-2-92420$ & $1-3$ & Electrical Installation & $\mathrm{S}$ & $\begin{array}{l}\text { Note: Important for } \\
\text { Maintenance }\end{array}$ \\
\hline $\mathrm{H}-2-92421$ & $1-4$ & Control Console Assembly & $\mathrm{S}$ & $\begin{array}{l}\text { Note: Important for } \\
\text { Maintenance }\end{array}$ \\
\hline H-2-92422 & $1-6$ & $\begin{array}{l}\text { Core Sample Truck \#1 Connection } \\
\text { Diagram }\end{array}$ & $\mathrm{E}$ & $\begin{array}{l}\text {-Depicts Equipment } \\
\text { Essential to Safe } \\
\text { Operation, -Depicts } \\
\text { Isolation Boundary for } \\
\text { Electrical or High-Pressure } \\
\text { Fluid Systems }\end{array}$ \\
\hline H-2-92432 & $1-2$ & $\begin{array}{l}\text { Shield Receiver Electrical } \\
\text { Installation }\end{array}$ & $S$ & $\begin{array}{l}\text { Note: Important for } \\
\text { Maintenance }\end{array}$ \\
\hline $\mathrm{H}-2-92433$ & 1 & Panel Assembly - Circuit Breaker & G & \\
\hline $\mathrm{H}-2-92434$ & 1 & Panel Detail -Circuit Breaker & G & \\
\hline
\end{tabular}


HNF-2305

Rev. 1

Table 4.1 Core Sample Truck 1 Drawings

\begin{tabular}{|c|c|l|c|l||}
\hline Dwg. No. & Sht. & \multicolumn{1}{|c|}{ Description } & Cat. & Justification/Notes \\
\hline \hline H-2-99726 & $1-2$ & $\begin{array}{l}\text { Shielded Receiver Tube } \\
\text { Replacement }\end{array}$ & G & \\
\hline H-2-827186 & $1-3$ & $\begin{array}{l}\text { Electrical Truck 1 Connection } \\
\text { Diagram }\end{array}$ & E & $\begin{array}{l}\text {-Depicts Electrical Power } \\
\text { Diagrams }\end{array}$ \\
\hline H-2-827190 & 1 & $\begin{array}{l}\text { Electrical Truck 1 One-Line } \\
\text { Diagram }\end{array}$ & E & -Depicts Electrical one-line \\
\hline
\end{tabular}

\subsubsection{Summary for Truck 1 Drawings}

Table 4.1.1 Summary for Truck 1 Drawings

\begin{tabular}{|l|c|c|}
\hline \hline \multicolumn{1}{|c|}{ Category } & Drawings & Sheets \\
\hline General Drawings & 28 & 40 \\
\hline Support Drawings. & $13^{*}$ & 44 \\
\hline Essential Drawings. & 5 & 14 \\
\hline
\end{tabular}

*Note: Drawing H-2-91701 which has both Support and Essential drawings sheets is included in the Essential drawing total. 
HNF-2305

Rev. 1

\subsection{CORE SAMPLE TRUCK 1 and 2 DRAWINGS}

Table 4.2 Core Sample Truck 1 \& 2 Drawings

\begin{tabular}{||c|c|l|c|l||}
\hline Dwg. & Sht. & \multicolumn{1}{|c|}{ Description } & Cat. & Justification/Notes \\
\hline \hline H-2-91428 & $1-2$ & $\begin{array}{l}\text { Shielded Receiver View Port } \\
\text { Assembly }\end{array}$ & G & \\
\hline H-2-91665 & $1-3$ & Remote Latch Unit Assembly & G & \\
\hline H-2-91666 & 1 & $\begin{array}{l}\text { 3" Ball Valve Modification } \\
\text { Assembly }\end{array}$ & G & \\
\hline H-2-91667 & 1 & Dust Cap and Sleeve Assembly & G & \\
\hline H-2-91668 & 1 & $\begin{array}{l}\text { Remote Latch Unit Weight } \\
\text { Assembly }\end{array}$ & G & Not Used \\
\hline H-2-91669 & 1 & Sampler Hoist Interlock Assembly & G & \\
\hline H-2-91702 & $1-3$ & $\begin{array}{l}\text { Core Sampler Truck Bed } \\
\text { Assembly }\end{array}$ & G & \\
\hline H-2-91703 & 1 & $\begin{array}{l}\text { Lower Frame \& Rotation Stop } \\
\text { Assembly }\end{array}$ & G & \\
\hline H-2-91704 & 1 & Lower Frame Assembly & G & \\
\hline H-2-91705 & 1 & Lower Bearing Mount Assembly & G & \\
\hline H-2-91706 & $1-2$ & Stationary Platform Assembly & G & \\
\hline H-2-91707 & $1-3$ & Rotating Platform Assembly & S & Note: Important for \\
Maintenance
\end{tabular}


HNF-2305

Rev. 1

Table 4.2 Core Sample Truck $1 \& 2$ Drawings

\begin{tabular}{||c|c|l|c|l||}
\hline \multicolumn{1}{|c|}{ Dwg. } & Sht. & \multicolumn{1}{|c|}{ Description } & Cat. & Justification/Notes \\
\hline H-2-91712 & 1 & Slide Rail Details & G & \\
\hline H-2-91713 & 1 & Control Console Support Frame & G & \\
\hline H-2-91715 & 1 & $\begin{array}{l}\text { Shielded Receiver Lifting Frame } \\
\text { Installation }\end{array}$ & G & \\
\hline H-2-91716 & $1-4$ & $\begin{array}{l}\text { Shielded Receiver Lifting Frame } \\
\text { Assembly }\end{array}$ & G & \\
\hline H-2-91717 & 1 & Air Compressor Frame Assembly & G & \\
\hline H-2-91718 & $1-2$ & Rear Jack Assembly & G & \\
\hline H-2-92423 & 1 & Motor Control Chassis Assembly & G & \\
\hline H-2-92424 & $1-2$ & $\begin{array}{l}\text { Panel and Control Chassis } \\
\text { Assembly }\end{array}$ & G & \\
\hline H-2-92427 & 1 & $\begin{array}{l}\text { Latching Control Chassis } \\
\text { Assembly }\end{array}$ & G & \\
\hline H-2-92428 & $1-2$ & Latching Control Chassis Details & G & \\
\hline H-2-92429 & 1 & Latch Control Panel Assembly & G & \\
\hline H-2-92430 & 1 & $\begin{array}{l}\text { Latch Control Circuit Board } \\
\text { Details }\end{array}$ & G & \\
\hline H-2-92431 & 1 & Latch Control Logic Diagram & S & \\
\hline H-2-92435 & 1 & Vent Panel Assembly & G & \\
\hline
\end{tabular}


HNF-2305

Rev. 1

\subsubsection{Summary for Truck $1 \& 2$ Drawings}

Table 4.2.1 Summary for Truck $1 \& 2$ Drawings

\begin{tabular}{|l|c|c|}
\hline \multicolumn{1}{|c|}{ Category } & Drawings & Sheets \\
\hline General Drawings & 28 & 46 \\
\hline Support Drawings. & 2 & 4 \\
\hline Essential Drawings. & 0 & 0 \\
\hline
\end{tabular}

\subsection{CORE SAMPLE TRUCK 2 DRAWINGS}

Table 4.3 Core Sample Truck 2 Drawings

\begin{tabular}{|c|c|l|c|l||}
\hline Dwg. & Sht. & \multicolumn{1}{|c|}{ Description } & Cat. & Justification/Notes \\
\hline \hline H-2-131570 & 1 & $\begin{array}{l}\text { Sampler Hoist Cable Drum } \\
\text { Assembly }\end{array}$ & G & On Standby \\
\hline H-2-140300 & $1-3$ & Core Sampler Truck 2 Assembly & G & On Standby \\
\hline H-2-140301 & $1-2$ & $\begin{array}{l}\text { Drawing Index Core Sampler } \\
\text { Truck 2 }\end{array}$ & G & On Standby \\
\hline H-2-140302 & $1-6$ & Drill Rig Equipment Installation & G & On Standby \\
\hline H-2-140303 & $1-6$ & Electrical Installation & G & On Standby \\
\hline H-2-140304 & $1-2$ & $\begin{array}{l}\text { Drill Rig Junction Box Enclosure } \\
\text { Assembly }\end{array}$ & G & On Standby \\
\hline H-2-140305 & $1-2$ & $\begin{array}{l}\text { Hydraulic Bottom Detector Alarm } \\
\text { Panel Assembly \& Details }\end{array}$ & G & On Standby \\
\hline H-2-140306 & 1 & Up Limit Switch Proximity Probe & G & On Standby \\
\hline H-2-140307 & $1-2$ & Aux Air Supply Installation & G & On Standby \\
\hline H-2-140308 & $1-3$ & Truck Frame Assembly & G & On Standby \\
\hline H-2-140309 & $1-2$ & Center Outrigger Jack Assembly & G & On Standby \\
\hline
\end{tabular}


HNF-2305

Rev. 1

Table 4.3 Core Sample Truck 2 Drawings

\begin{tabular}{|l|c|l|c|l||}
\hline \multicolumn{1}{|c|}{ Dwg. } & Sht. & \multicolumn{1}{|c|}{ Description } & Cat. & Justification/Notes \\
\hline H-2-81785 & $1-17$ & $\begin{array}{l}\text { Core Sample Truck \#2 Electrical } \\
\text { Connection Diagram }\end{array}$ & G & On Standby \\
\hline H-2-81834 & $1-5$ & Grapple Hoist Installation & G & On Standby \\
\hline H-2-81835 & $1-2$ & $\begin{array}{l}\text { Grapple Hoist Mounting Support } \\
\text { Assembly }\end{array}$ & G & On Standby \\
\hline H-2-81836 & $1-6$ & Grapple Hoist Assembly & G & On Standby \\
\hline H-2-81837 & $1-2$ & Grapple Hoist Housing Assembly & G & On Standby \\
\hline H-2-81838 & $1-4$ & Purge Gas Enclosure Assembly & G & On Standby \\
\hline H-2-81839 & $1-5$ & Purge Gas Piping Assembly & G & On Standby \\
\hline H-2-81840 & $1-3$ & Control Console Assembly & G & On Standby \\
\hline H-2-81841 & $1-6$ & $\begin{array}{l}\text { Purge Gas Piping and Hose } \\
\text { Installation }\end{array}$ & G & On Standby \\
\hline H-2-81844 & $1-3$ & Quill Rod Assembly & G & Not used \\
\hline H-2-81845 & $1-2$ & Kamlock Adapter Assembly & G & On Standby \\
\hline H-2-81846 & $1-3$ & Hydraulic Reservoir Assembly & G & On Standby \\
\hline H-2-81848 & $1-2$ & $\begin{array}{l}\text { Grapple Hoist Rotary Valve } \\
\text { Actuator Assembly }\end{array}$ & G & On Standby \\
\hline H-2-81849 & 1 & Remote Latch Unit Weight Assy & G & On Standby \\
\hline H-2-81850 & $1-8$ & Hydraulic Controls Installation & G & On Standby \\
\hline H-2-81851 & 1 & Cable Connection Diagram & G & On Standby \\
\hline H-2-81852 & 1 & $\begin{array}{l}\text { Overall Electrical Power } \\
\text { Distribution Diagram }\end{array}$ & G & $\begin{array}{l}\text { Duplicates information on } \\
\text { H-2-827187 On Standby }\end{array}$ \\
\hline H-2-81854 & 1 & Loadcell Readout Assembly & G & On Standby \\
\hline H-2-81855 & $1-2$ & Front Jack Assembly & G & On Standby \\
\hline
\end{tabular}


HNF-2305

Rev. 1

Table 4.3 Core Sample Truck 2 Drawings

\begin{tabular}{||c|c|l|c|l||}
\hline Dwg. & Sht. & \multicolumn{1}{|c|}{ Description } & Cat. & Justification/Notes \\
\hline H-2-82102 & $1-3$ & $\begin{array}{l}\text { Line Conditioner \& Control Select } \\
\text { Panel Assembly }\end{array}$ & G & On Standby \\
\hline H-2-82103 & 1 & $\begin{array}{l}\text { Line Conditioner \& Control Select } \\
\text { Panel Installation }\end{array}$ & G & On Standby \\
\hline H-2-821454 & $1-4$ & Breathing Air Piping Installation & G & $\begin{array}{l}\text { Note: Installed but not } \\
\text { used On Standby }\end{array}$ \\
\hline H-2-83111 & $1-7$ & $\begin{array}{l}\text { Core Sampler Truck \#2 } \\
\text { Elementary Diagrams }\end{array}$ & G & On Standby \\
\hline H-2-83112 & $1-6$ & Instrument Enclosure Assembly & G & On Standby \\
\hline H-2-83115 & $1-4$ & Purge Gas Assembly & G & On Standby \\
\hline H-2-83307 & $1-5$ & Sampler Hoist Assembly & G & On Standby \\
\hline H-2-83308 & $1-3$ & Sampler Hoist Housing Assembly & G & On Standby \\
\hline H-2-83309 & $1-2$ & Shielded Receiver Base Assembly & G & On Standby \\
\hline H-2-83310 & $1-2$ & Sampler Hoist Load Stand & G & On Standby \\
\hline H-2-83314 & $1-4$ & Shielded Receiver Assembly & G & In Standby \\
\hline H-2-83315 & $1-2$ & $\begin{array}{l}\text { Shielded Receiver Electrical } \\
\text { Assembly }\end{array}$ & G & On Standby \\
\hline H-2-83316 & $1-3$ & $\begin{array}{l}\text { Shielded Receiver Weather Cover } \\
\text { Assembly }\end{array}$ & G & On Standby \\
\hline H-2-83891 & $1-3$ & Hydraulic Flow Diagram & G & $\begin{array}{l}\text {-Depicts Process Diagrams } \\
\text { On Standby }\end{array}$ \\
\hline H-2-85068 & $1-2$ & Purge Gas Pneumatic Diagram & G & $\begin{array}{l}\text {-Depicts High Pressure } \\
\text { Diagram On Standby }\end{array}$ \\
\hline H-2-89435 & $1-4$ & $\begin{array}{l}\text { Drillhead Service Platform } \\
\text { Installation }\end{array}$ & G & On Standby \\
\hline
\end{tabular}


HNF-2305

Rev. 1

Table 4.3 Core Sample Truck 2 Drawings

\begin{tabular}{||c|c|l|c|l||}
\hline \multicolumn{1}{|c|}{ Dwg. } & Sht. & \multicolumn{1}{c|}{ Description } & Cat. & Justification/Notes \\
\hline H-2-89436 & $1-3$ & $\begin{array}{l}\text { Drillhead Service Platform } \\
\text { Assembly }\end{array}$ & G & On Standby \\
\hline H-2-89437 & $1-2$ & Rpm Sensing Ring Installation & G & On Standby \\
\hline H-2-89438 & $1-4$ & Penetration Rate Assembly & G & On Standby \\
\hline H-2-89439 & $1-5$ & $\begin{array}{l}\text { Drillhead Service Platform } \\
\text { Assembly }\end{array}$ & G & On Standby \\
\hline H-2-89440 & $1-2$ & Pressure Indicator Light Assembly & G & On Standby \\
\hline H-2-89441 & 1 & Circuit Breaker Panel Detail & G & On Standby \\
\hline H-2-89442 & $1-3$ & Drillhead Hydraulic Installation & G & On Standby \\
\hline H-2-89443 & $1-5$ & Inner/Outer Bellows Assembly & G & On Standby \\
\hline H-2-89444 & 1 & Cable Wiper Assembly & G & On Standby \\
\hline H-2-89459 & $1-13$ & Alarm \& Control Diagram & G & $\begin{array}{l}\text {-Depicts Equipment } \\
\text { Essential to Safe Operation } \\
\text {-Depicts \& Identifies } \\
\text { Instrumentation Required } \\
\text { by AB On Standby }\end{array}$ \\
\hline H-2-827191 & 1 & $\begin{array}{l}\text { Electrical Truck 2 One Line } \\
\text { Diagram }\end{array}$ & G & $\begin{array}{l}\text {-Depicts Electrical One- } \\
\text { line Diagram On Standby }\end{array}$ \\
\hline H-2-89462 & $1-3$ & Purge Gas Connection Box & G & On Standby \\
\hline H-2-89463 & $1-2$ & Purge Gas Cable Assembly & G & On Standby \\
\hline H-2-827187 & $1-8$ & $\begin{array}{l}\text { Electrical Truck 2 Connection } \\
\text { Diagram }\end{array}$ & G & $\begin{array}{l}\text { - Depicts Electrical Power } \\
\text { Diagram On Standby }\end{array}$ \\
\hline
\end{tabular}


HNF-2305

Rev. 1

\subsubsection{Summary of Core Sample Truck 2}

Table 4.3.1 Summary for Truck 2 Drawings

\begin{tabular}{|l|c|c|}
\hline \multicolumn{1}{|c|}{ Category } & Drawings & Sheets \\
\hline \hline General Drawings & 60 & 212 \\
\hline Support Drawings. & 0 & 0 \\
\hline Essential Drawings. & 0 & 0 \\
\hline
\end{tabular}

\subsection{CORE SAMPLE TRUCK 3 \& 4 DRAWINGS}

Table 4.4 RMCST 3 \& 4 Drawings

\begin{tabular}{||c|c|l|c|l||}
\hline \multicolumn{1}{|c|}{ Dwg. } & Sht. & \multicolumn{1}{|c|}{ Description } & Cat. & Justification/Notes \\
\hline \hline H-2-690000 & $1-2$ & RMCST Truck 3 \& 4 Assembly & S & \\
\hline H-2-690001 & $1-2$ & $\begin{array}{l}\text { RMCST Truck 3 \& 4 Drawing } \\
\text { Index }\end{array}$ & S & $\begin{array}{l}\text { Note: Important for } \\
\text { Maintenance }\end{array}$ \\
\hline H-2-690005 & $1-5$ & Drill Rig Equipment Installation & G & \\
\hline H-2-690006 & $1-3$ & Hydraulic Reservoir Assembly & G & \\
\hline H-2-690007 & $1-2$ & Pressure Indicator Light Assembly & G & \\
\hline H-2-690008 & $1-3$ & Hydraulic Flow Diagram & E & -Depicts Process Diagrams \\
\hline H-2-690009 & $1-2$ & Purge Gas Pneumatic Diagram & E & $\begin{array}{l}\text {-Depicts High Pressure } \\
\text { Process \& Instrumentation } \\
\text { Diagram }\end{array}$ \\
\hline H-2-690010 & $1-3$ & Truck Frame Assembly & G & \\
\hline H-2-690011 & $1-2$ & Center Outrigger Jack Assembly & G & \\
\hline H-2-690012 & $1-6$ & $\begin{array}{l}\text { Purge Gas Piping \& Hose } \\
\text { Installation }\end{array}$ & S & $\begin{array}{l}\text { Note: Important for } \\
\text { Maintenance }\end{array}$ \\
\hline H-2-690013 & $1-2$ & Auxiliary Air Supply Installation & G & \\
\hline
\end{tabular}


HNF-2305

Rev. 1

Table 4.4 RMCST 3 \& 4 Drawings

\begin{tabular}{||c|c|l|c|l||}
\hline Dwg. & Sht. & \multicolumn{1}{|c|}{ Description } & Cat. & Justification/Notes \\
\hline \hline H-2-690014 & $1-3$ & $\begin{array}{l}\text { Drill Head Hydraulic Piping } \\
\text { Installation }\end{array}$ & S & $\begin{array}{l}\text { Note: Important for } \\
\text { Maintenance }\end{array}$ \\
\hline H-2-690015 & $1-8$ & Hydraulic Controls Installation & S & $\begin{array}{l}\text { Note: Important for } \\
\text { Maintenance }\end{array}$ \\
\hline H-2-690016 & $1-3$ & Intercom System Installation & G & \\
\hline H-2-690017 & $1-2$ & Rear Jack Assembly & G & \\
\hline H-2-690020 & $1-4$ & Shielded Receiver Assembly & S & $\begin{array}{l}\text { Note: Important for } \\
\text { Maintenance }\end{array}$ \\
\hline H-2-690021 & $1-3$ & $\begin{array}{l}\text { Line Conditioner \& Control } \\
\text { Electrical Panel Assembly }\end{array}$ & G & \\
\hline H-2-690022 & 1 & Shielded Receiver Tube Assembly & G & \\
\hline H-2-690023 & 1 & 3" Ball Valve Modification" & G & \\
\hline H-2-690024 & 1 & Dust Cap and Sleeve Assembly & G & \\
\hline H-2-690025 & $1-2$ & View Port Shielded Receiver & G & \\
\hline H-2-690026 & $1-2$ & $\begin{array}{l}\text { Shielded Receiver Electrical } \\
\text { Installation }\end{array}$ & S & $\begin{array}{l}\text { Note: Important for } \\
\text { Maintenance }\end{array}$ \\
\hline H-2-690027 & $1-2$ & Shielded Receiver Base Assembly & G & \\
\hline H-2-690028 & $1-6$ & Shielded Receiver Weather Cover & G & \\
\hline H-2-690030 & $1-4$ & Sampler Hoist Assembly & S & $\begin{array}{l}\text { Note: Important for } \\
\text { Maintenance }\end{array}$ \\
\hline H-2-690031 & $1-3$ & $\begin{array}{l}\text { Sampler Hoist Cable Feed } \\
\text { Assembly }\end{array}$ & G & \\
\hline H-2-690032 & 1 & $\begin{array}{l}\text { Sampler Hoist Cable Guide } \\
\text { Assembly }\end{array}$ & G & \\
\hline H-2-690033 & $1-2$ & $\begin{array}{l}\text { Sampler Hoist Load Stand } \\
\text { Assembly }\end{array}$ & G & \\
\hline & & & \\
\hline
\end{tabular}


HNF-2305

Rev. 1

Table 4.4 RMCST 3 \& 4 Drawings

\begin{tabular}{|c|c|c|c|c|}
\hline Dwg. & Sht. & Description & Cat. & Justification/Notes \\
\hline $\mathrm{H}-2-690034$ & $1-4$ & Sampler Hoist Housing Assembly & G & \\
\hline H-2-690035 & $1-4$ & Breather Air Piping Installation & G & $\begin{array}{l}\text { Note: Installed but not } \\
\text { Used }\end{array}$ \\
\hline H-2-690036 & $1-2$ & $\begin{array}{l}\text { Sampler Hoist Cable Drum } \\
\text { Assembly }\end{array}$ & G & \\
\hline H-2-690037 & 1 & Sampler Hoist Interlock Assembly & G & \\
\hline H-2-690039 & 1 & $\begin{array}{l}\text { Remote Latch Unit Weight } \\
\text { Assembly }\end{array}$ & G & \\
\hline H-2-690040 & $1-5$ & RMCST Truck Bed Assembly & G & \\
\hline H-2-690041 & $1-4$ & $\begin{array}{l}\text { Rotary \& Stationary Platform Rail } \\
\text { Assembly }\end{array}$ & G & \\
\hline $\mathrm{H}-2-690042$ & $1-2$ & Stationary Platform Assembly & G & \\
\hline H-2-690043 & 1 & $\begin{array}{l}\text { Shielded Receiver Lifting Frame } \\
\text { Installation }\end{array}$ & G & \\
\hline H-2-690044 & $1-2$ & $\begin{array}{l}\text { Shielded Receiver Lifting Frame } \\
\text { Assembly }\end{array}$ & G & \\
\hline H-2-690046 & 1 & Lower Frame Assembly & G & \\
\hline H-2-690048 & 1 & Lower Bearing Mount Assembly & G & \\
\hline H-2-690049 & 1 & $\begin{array}{l}\text { Lower Frame \& Rotary Stop } \\
\text { Assembly }\end{array}$ & G & \\
\hline $\mathrm{H}-2-690050$ & $1-5$ & $\begin{array}{l}\text { Drillhead Service Platform } \\
\text { Installation }\end{array}$ & G & \\
\hline $\mathrm{H}-2-690051$ & $1-3$ & Quill Rod Assembly & G & Used as spare for Truck 2 \\
\hline $\mathrm{H}-2-690052$ & $1-2$ & Grapple Rotary Valve Actuator & G & \\
\hline H-2-690053 & $1-4$ & Penetration Rate Assembly & G & \\
\hline H-2-690054 & $1-2$ & Rpm Sensing Ring Installation & G & \\
\hline
\end{tabular}


HNF-2305

Rev. 1

Table 4.4 RMCST 3 \& 4 Drawings

\begin{tabular}{||c|c|l|c|l||}
\hline Dwg. & Sht. & \multicolumn{1}{|c|}{ Description } & Cat. & Justification/Notes \\
\hline \hline H-2-690055 & $1-5$ & Grapple Hoist Installation & S & $\begin{array}{l}\text { Note: Important for } \\
\text { Maintenance }\end{array}$ \\
\hline H-2-690056 & $1-2$ & Grapple Hoist Mounting Support & G & \\
\hline H-2-690057 & $1-7$ & Grapple Hoist Assembly & S & $\begin{array}{l}\text { Note: Important for } \\
\text { Maintenance }\end{array}$ \\
\hline H-2-690058 & $1-2$ & Grapple Hoist Housing Assembly & G & \\
\hline H-2-690059 & $1-6$ & Inner/Outer Bellows Assembly & S & $\begin{array}{l}\text { Note: Important for } \\
\text { Maintenance }\end{array}$ \\
\hline H-2-690060 & $1-4$ & Purge Gas Assembly & S & $\begin{array}{l}\text { Note: Important for } \\
\text { Maintenance }\end{array}$ \\
\hline H-2-690061 & $1-4$ & Purge Gas Enclosure Assembly & G & \\
\hline H-2-690062 & $1-5$ & Purge Gas Piping Assembly & S & $\begin{array}{l}\text { Note: Important for } \\
\text { Maintenance }\end{array}$ \\
\hline H-2-690063 & $1-3$ & Purge Gas Connection Box & G & \\
\hline H-2-690064 & $1-2$ & Purge Gas Cable Assembly & G & \\
\hline H-2-690065 & 1 & Up Limit Switch Proximity Probe & G & \\
\hline H-2-690066 & $1-3$ & $\begin{array}{l}\text { Drillhead Service Platform } \\
\text { Assembly }\end{array}$ & G & \\
\hline H-2-690067 & $1-5$ & Drillhead Service Platform Assy & G & \\
\hline H-2-690068 & $1-7$ & Instrument Enclosure Assembly & S & $\begin{array}{l}\text { Note: Important for } \\
\text { Maintenance }\end{array}$ \\
\hline H-2-690069 & $1-18$ & Alarm \& Control Diagram & E & $\begin{array}{l}\text {-Depicts Required Alarm } \\
\text { Systems and Equipment } \\
\text { Essential to Safe Operation }\end{array}$ \\
\hline H-2-690070 & $1-7$ & Elementary Diagram & E & $\begin{array}{l}\text {-Depicts Equipment } \\
\text { Essential to Safe Operation }\end{array}$ \\
\hline
\end{tabular}


HNF-2305

Rev. 1

Table 4.4 RMCST 3 \& 4 Drawings

\begin{tabular}{||c|c|l|c|l||}
\hline \multicolumn{1}{|c|}{ Dwg. } & Sht. & \multicolumn{1}{|c|}{ Description } & Cat. & Justification/Notes \\
\hline \hline H-2-690071 & $1-17$ & Electrical Connection Diagram & S & $\begin{array}{l}\text { Note: Important for } \\
\text { Maintenance }\end{array}$ \\
\hline H-2-690073 & $1-7$ & Electrical Installation & S & $\begin{array}{l}\text { Note: Important for } \\
\text { Maintenance }\end{array}$ \\
\hline H-2-690074 & $1-2$ & $\begin{array}{l}\text { Hydraulic Bottom Detector Alarm } \\
\text { Panel Assembly }\end{array}$ & G & \\
\hline H-2-690076 & $1-2$ & $\begin{array}{l}\text { Drill Rig Junction Box Enclosure } \\
\text { Assembly }\end{array}$ & G & \\
\hline H-2-690080 & $1-3$ & Rotary Platform Assembly & S & $\begin{array}{l}\text { Note: Important for } \\
\text { Maintenance }\end{array}$ \\
\hline H-2-690081 & $1-2$ & Rotary Platform Weldment & G & \\
\hline H-2-690082 & 1 & Bearing Plate Assembly & G & \\
\hline H-2-690083 & 1 & Rotary Stop Assembly & G & \\
\hline H-2-690084 & 1 & Slide Rail Details & G & \\
\hline H-2-690085 & 1 & $\begin{array}{l}\text { Control Console Support Frame } \\
\text { Assembly }\end{array}$ & G & \\
\hline H-2-690090 & $1-3$ & Control Console Assembly & G & \\
\hline H-2-690092 & $1-3$ & Motor Control Chassis Assembly & G & \\
\hline H-2-690094 & $1-2$ & Encoder Panel Assembly & G & \\
\hline H-2-690095 & $1-2$ & Loadcell Readout Assembly & G & \\
\hline H-2-690096 & $1-2$ & Hydraulic \& Sampler Actuator & G & \\
\hline H-2-690097 & 1 & Panel Detail Circuit Breaker & G & \\
\hline H-2-690098 & 1 & Vent Panel Assembly & G & \\
\hline H-2-690106 & $1-3$ & Front Jack Assembly & G & \\
\hline
\end{tabular}


HNF-2305

Rev. 1

Table 4.4 RMCST 3 \& 4 Drawings

\begin{tabular}{||c|c|l|c|l||}
\hline Dwg. & Sht. & \multicolumn{1}{|c|}{ Description } & Cat. & Justification/Notes \\
\hline H-2-690143 & $1-2$ & Mechanical Remote Latch Unit & S & $\begin{array}{l}\text { Note: Important for } \\
\text { Maintenance }\end{array}$ \\
\hline H-2-827188 & $1-9$ & $\begin{array}{l}\text { Electrical Truck 3 Connection } \\
\text { Diagram }\end{array}$ & E & $\begin{array}{l}\text {-Depicts Electrical Power } \\
\text { Diagrams }\end{array}$ \\
\hline H-2-827189 & $1-7$ & $\begin{array}{l}\text { Electrical Truck 4 Connection } \\
\text { Diagram }\end{array}$ & E & $\begin{array}{l}\text {-Depicts Electrical Power } \\
\text { Diagrams }\end{array}$ \\
\hline H-2-827192 & $1-2$ & $\begin{array}{l}\text { Electrical Truck 3 One Line } \\
\text { Diagram }\end{array}$ & E & $\begin{array}{l}\text {-Depicts Electrical One- } \\
\text { line }\end{array}$ \\
\hline H-2-827193 & $1-2$ & $\begin{array}{l}\text { Electrical Truck 4 One Line } \\
\text { Diagram }\end{array}$ & E & $\begin{array}{l}\text {-Depicts Electrical One- } \\
\text { line }\end{array}$ \\
\hline
\end{tabular}

\subsubsection{Summary RMCST 3 \& 4 Drawings}

Table 4.4.1 Summary RMCST 3 \& 4 Drawings

\begin{tabular}{|l|c|c|}
\hline \hline \multicolumn{1}{|c|}{ Category } & Drawings & Sheets \\
\hline General Drawings & 59 & 138 \\
\hline Support Drawings. & 18 & 94 \\
\hline Essential Drawings. & 8 & 50 \\
\hline
\end{tabular}

\subsection{CORE SAMPLING SUPPORT EQUIPMENT DRAWINGS}

Table 4.5 Core Sampling Support Equipment Drawings

\begin{tabular}{|c|c|l|c|l||}
\hline Dwg. & Sht. & \multicolumn{1}{|c|}{ Description } & Cat. & Justification/Notes \\
\hline H-2-34727 & 1 & Core Sampling Cask & G & Not Used \\
\hline
\end{tabular}


HNF-2305

Rev. 1

Table 4.5 Core Sampling Support Equipment Drawings

\begin{tabular}{||c|c|l|c|l||}
\hline \multicolumn{1}{|c|}{ Dwg. } & Sht. & \multicolumn{1}{|c|}{ Description } & Cat. & Justification/Notes \\
\hline \hline H-2-34994 & 1 & $\begin{array}{l}\text { Core Sampling Shipping Cask } \\
\text { Arrangement }\end{array}$ & G & Not Used \\
\hline H-2-34995 & 1 & $\begin{array}{l}\text { Core Sampling Shipping Cask } \\
\text { Detail }\end{array}$ & G & Not Used \\
\hline H-2-34996 & 1 & Core Sampling Shipping Cask & G & Not Used \\
\hline H-2-36057 & 1 & $\begin{array}{l}\text { Core Sampling Flapper } \\
\text { Assembly }\end{array}$ & G & Not Used \\
\hline H-2-36511 & 1 & Core Drill Pipe Fitting & G & Not Used \\
\hline H-2-36514 & 1 & Core Sampling Seal & G & Not Used \\
\hline H-2-36766 & 1 & $\begin{array}{l}\text { Core Sampling Equipment } \\
\text { Arrangement }\end{array}$ & G & Not Used \\
\hline H-2-36769 & 1 & Core Sampling 12-in Flange & G & Not Used \\
\hline H-2-38078 & $1-2$ & On-site Transfer Cask Stand & S & \\
\hline H-2-38079 & $1-6$ & On-site Transfer Cask & S & \\
\hline H-2-38191 & 1 & $\begin{array}{l}\text { Arrangement and Detail Core } \\
\text { Sampler and Drill }\end{array}$ & G & \\
\hline H-2-38192 & 1 & $\begin{array}{l}\text { Assembly and Detail Adapter } \\
\text { Flange Spray Washer Spray }\end{array}$ & G & \\
\hline H-2-38193 & 1 & $\begin{array}{l}\text { Portable Core Sampling } \\
\text { Platform }\end{array}$ & G & \\
\hline H-2-38194 & 1 & Drip Pan for Core Sample & G & \\
\hline H-2-38195 & 1 & $\begin{array}{l}\text { Commercial Drill Equipment } \\
\text { Mod }\end{array}$ & G & \\
\hline H-2-38196 & 1 & $\begin{array}{l}\text { Misc Detail and Arrangement } \\
\text { for Core Sampling }\end{array}$ & G & \\
\hline H-2-74814 & $1-5$ & Cask Truck & G & \\
\hline
\end{tabular}


HNF-2305

Rev. 1

Table 4.5 Core Sampling Support Equipment Drawings

\begin{tabular}{|l|c|l|c|l||}
\hline \multicolumn{1}{|c|}{ Dwg. } & Sht. & \multicolumn{1}{c|}{ Description } & Cat. & Justification/Notes \\
\hline H-2-74815 & 1 & Cask Truck & G & \\
\hline H-2-74816 & 1 & Cask Truck & G & \\
\hline H-2-74819 & 1 & Cask Truck & G & \\
\hline H-2-74820 & 1 & Cask Truck & G & \\
\hline H-2-75053 & 1 & $\begin{array}{l}\text { Sample Truck Pig Transport } \\
\text { Rack Assy }\end{array}$ & G & \\
\hline H-2-79840 & 1 & Service Trailer Truck 1 & G & Not Used \\
\hline H-2-79841 & $1-3$ & Service Trailer Truck 1 & G & Not Used \\
\hline H-2-79842 & $1-2$ & Service Trailer Truck 2 & G & \\
\hline H-2-79843 & $1-2$ & Service Trailer Truck 2 & G & \\
\hline H-2-81842 & $1-2$ & Cable Spray Washer Assy & G & \\
\hline H-2-81843 & 1 & Cable Spray Washer Housing & G & \\
\hline H-2-81853 & $1-6$ & Support Truck & G & \\
\hline H-2-83319 & $1-2$ & Cask Truck, Unit 1 & G & \\
\hline H-2-83320 & $1-4$ & Cask Truck, Unit 1 & G & \\
\hline H-2-83323 & $1-2$ & Cask Truck Detail, Unit 2 & G & \\
\hline H-2-83324 & $1-4$ & Unit 2 Cask Truck & G & \\
\hline H-2-83325 & $1-5$ & Unit 1 Support Truck & G & \\
\hline H-2-85064 & $1-3$ & $\begin{array}{l}\text { Sampler Valve Housing } \\
\text { Removal Tool }\end{array}$ & G & Used in Lab \\
\hline H-2-85066 & $1-2$ & Changeout Valve & G & Not Used \\
\hline H-2-85287 & $1-3$ & Mobile Air Charger Assembly & G & \\
\hline H-2-85299 & $1-7$ & $\begin{array}{l}\text { Nitrogen Trailer Hose Reel } \\
\text { Installation }\end{array}$ & G & \\
\hline & & & \\
\hline
\end{tabular}


HNF-2305

Rev. 1

Table 4.5 Core Sampling Support Equipment Drawings

\begin{tabular}{|c|c|c|c|c|}
\hline Dwg. & Sht. & Description & Cat. & Justification/Notes \\
\hline$H-285299$ & $8-10$ & $\begin{array}{l}\text { Nitrogen Trailer Hose Reel } \\
\text { Installation }\end{array}$ & $E$ & -P \& ID For $\mathrm{N}_{2}$ Trailer \\
\hline $\mathrm{H}-2-85327$ & 4 & $\begin{array}{l}\text { Panelboard Schedule, Electrical } \\
\text { Distribution Trailer \#1 }\end{array}$ & $\mathrm{E}$ & $\begin{array}{l}\text { Already Essential (Other } \\
\text { sheets of this drawing are } \\
\text { NOT for sampling } \\
\text { equipment.) }\end{array}$ \\
\hline $\mathrm{H}-2-85327$ & 5 & $\begin{array}{l}\text { Panelboard Schedule, Electrical } \\
\text { Distribution Trailer \#2 }\end{array}$ & $\mathrm{E}$ & $\begin{array}{l}\text { Already Essential (Other } \\
\text { sheets of this drawing are } \\
\text { NOT for sampling } \\
\text { equipment.) }\end{array}$ \\
\hline $\mathrm{H}-2-85340$ & 1 & $\begin{array}{l}\text { Distribution Trailer One-Line } \\
\text { Diagram }\end{array}$ & $\mathrm{E}$ & $\begin{array}{l}\text {-Depicts Electrical One- } \\
\text { line }\end{array}$ \\
\hline $\mathrm{H}-2-85341$ & $1-2$ & Electrical Distribution Trailer \#2 & G & \\
\hline H-2-85342 & 1 & Electrical Distribution Trailer \#1 & G & \\
\hline $\mathrm{H}-2-85345$ & 1 & Core Barrel Assembly & G & Not Used \\
\hline $\mathrm{H}-2-85349$ & $1-3$ & Riser Adapters Assorted & G & \\
\hline $\mathrm{H}-2-85350$ & $1-2$ & $\begin{array}{l}\text { Sampling Electrical Distribution. } \\
\text { Trailer }\end{array}$ & G & \\
\hline H-2-85358 & $1-4$ & Portable Platform Assembly & G & \\
\hline H-2-85601 & $1-2$ & $\begin{array}{l}\text { Breathing Air Compressor } \\
\text { Electrical One-Line Diagram }\end{array}$ & $\mathrm{E}$ & \\
\hline $\mathrm{H}-2-85633$ & $1-4$ & $\begin{array}{l}\text { Structural Truck Ramp Platform } \\
\text { Arrangement }\end{array}$ & G & \\
\hline $\mathrm{H}-2-90257$ & 1 & Core Sampler Latch Mechanism & $\mathrm{G}$ & Not Used \\
\hline H-2-91291 & $1-5$ & Sampler Hot Cell Unloader & G & Not Used \\
\hline $\mathrm{H}-2-91330$ & 1 & $\begin{array}{l}\text { Pull Rod Magnetic Retrieval } \\
\text { Tool }\end{array}$ & G & \\
\hline
\end{tabular}


HNF-2305

Rev. 1

Table 4.5 Core Sampling Support Equipment Drawings

\begin{tabular}{||l|c|l|c|l||}
\hline \multicolumn{1}{|c|}{ Dwg. } & Sht. & \multicolumn{1}{c|}{ Description } & Cat. & Justification/Notes \\
\hline H-2-91331 & 1 & Magnetic Positioning Tools & G & \\
\hline H-2-91398 & 1 & Sampler Hot Cell Unloader & G & Not Used \\
\hline H-2-91422 & $1-2$ & Sampler Shoe Assembly & G & Not Used \\
\hline H-2-91425 & $1-2$ & Drill Bit Modification & G & Not Used \\
\hline H-2-91426 & 1 & Drill Bit Modification & G & Not Used \\
\hline H-2-91427 & $1-2$ & Drill Bit Plug Remover & G & Not Used \\
\hline H-2-91490 & $1-5$ & Sampler Hot Cell Unloader & G & Not Used \\
\hline H-2-91497 & $1-3$ & $\begin{array}{l}\text { Core Sampler Assembly and } \\
\text { Detail }\end{array}$ & G & Not Used \\
\hline H-2-91498 & $1-3$ & $\begin{array}{l}\text { Core Sampler Assembly and } \\
\text { Detail }\end{array}$ & G & Not Used \\
\hline H-2-91502 & 1 & $\begin{array}{l}\text { Core Catcher Assembly and } \\
\text { Detail }\end{array}$ & G & Not Used \\
\hline H-2-91503 & $1-3$ & $\begin{array}{l}\text { Core Catcher Assembly and } \\
\text { Detail }\end{array}$ & G & Not Used \\
\hline H-2-91526 & 1 & 12" Tank Riser Arrangement & G & Not Used \\
\hline H-2-91527 & $1-2$ & 12" Drill Rod Guide & G & Not Used \\
\hline H-2-91539 & 1 & 8 " Tank Riser Arrangement & G & Not Used \\
\hline H-2-91540 & $1-2$ & 8 " Drill Rod Guide & G & Not Used \\
\hline H-2-91544 & 1 & 6" Tank Riser Arrangement & G & Not Used \\
\hline H-2-91545 & $1-2$ & 6" Drill Rod Guide & G & Not Used \\
\hline H-2-91546 & 1 & $\begin{array}{l}\text { 12" X 6" Riser Adapter } \\
\text { Assembly }\end{array}$ & G & Not Used \\
\hline H-2-91549 & 1 & 4" Tank Riser Arrangement & G & \\
\hline
\end{tabular}


HNF-2305

Rev. 1

Table 4.5 Core Sampling Support Equipment Drawings

\begin{tabular}{|c|c|c|c|c|}
\hline Dwg. & Sht. & Description & Cat. & Justification/Notes \\
\hline $\mathrm{H}-2-91550$ & $1-2$ & 4" Drill Rod Guide & G & Not Used \\
\hline H-2-91551 & 1 & 12" X 4" Riser Adapter & G & Not Used \\
\hline H-2-91570 & $1-2$ & Hot Cell Sample Removal Tool & $\mathrm{G}$ & Not Used \\
\hline H-2-91574 & $1-3$ & Drill String Arrangement & G & \\
\hline H-2-91575 & 1 & Core Barrel Assembly & G & Not Used \\
\hline H-2-91670 & 1 & $\begin{array}{l}\text { Drill Rodwasher Housing } \\
\text { Assembly and Details }\end{array}$ & G & \\
\hline H-2-91671 & $1-3$ & $\begin{array}{l}\text { Drill Rodwasher Manifold } \\
\text { Assembly and Details }\end{array}$ & G & \\
\hline H-2-91672 & 1 & Cover Plate Assembly & G & \\
\hline H-2-91673 & 1 & $\begin{array}{l}12^{\prime \prime} \mathrm{X} 18^{\prime \prime} \text { Riser Adapter } \\
\text { Assembly and Details }\end{array}$ & G & \\
\hline H-2-91674 & $1-3$ & $\begin{array}{l}\text { Transport Cask Stand Assembly } \\
\text { and Details }\end{array}$ & G & \\
\hline H-2-91675 & 1 & $\begin{array}{l}\text { Cask Adapter Assembly and } \\
\text { Details }\end{array}$ & G & \\
\hline H-2-91676 & 1 & PVC Sleeve Details & G & Not Used \\
\hline H-2-91677 & $1-2$ & $\begin{array}{l}\text { Kamlock Adapter Assembly } \\
\text { Core Sampler Truck \#1 }\end{array}$ & G & . \\
\hline H-2-91678 & 1 & Dust Cap Assembly & G & \\
\hline H-2-91684 & 1 & Modified Quadralatch & G & Not Used \\
\hline H-2-91687 & 1 & $\begin{array}{l}\text { Reusable Pull Rod Overpack } \\
\text { Assembly }\end{array}$ & G & Not Used \\
\hline H-2-94558 & 1 & Sample Truck Hoist Trolley & $\mathrm{G}$ & \\
\hline H-2-99332 & 1 & $12^{\prime \prime}$ X 4" Riser Adapter & G & \\
\hline
\end{tabular}


HNF-2305

Rev. 1

Table 4.5 Core Sampling Support Equipment Drawings

\begin{tabular}{|l|c|l|c|l||}
\hline \multicolumn{1}{|c|}{ Dwg. } & Sht. & \multicolumn{1}{|c|}{ Description } & Cat. & Justification/Notes \\
\hline \hline H-2-99333 & 1 & 12" X 6" Riser Adapter & G & \\
\hline H-2-99334 & 1 & $12^{\prime \prime}$ X 8" Riser Adapter & G & \\
\hline H-2-99335 & 1 & $12^{\prime \prime}$ X 4" Riser Adapter & G & \\
\hline H-2-99336 & 1 & $\begin{array}{l}6,8 \text { \& 4" X 4" Offset Riser } \\
\text { Adapter }\end{array}$ & G & \\
\hline H-2-99346 & 1 & $\begin{array}{l}4,6, \text { and 8" Waste Tank Riser } \\
\text { Arrangement }\end{array}$ & G & \\
\hline H-2-99347 & 1 & $\begin{array}{l}\text { 6,8, 10, \& 12" Offset Riser } \\
\text { Adapter }\end{array}$ & G & \\
\hline H-2-99725 & $1-5$ & Transfer Cask-NCAW & S & \\
\hline H-2-99727 & $1-2$ & Ebr-2 Storage Cask Modified & G & \\
\hline H-2-99729 & 1 & $\begin{array}{l}\text { Ebr-2 Cask/shield Receiver } \\
\text { Adapter }\end{array}$ & G & \\
\hline H-2-99730 & 1 & Drill String Burial Container & G & \\
\hline H-2-99731 & $1-2$ & $\begin{array}{l}\text { NCAW Transport Cask Lifting } \\
\text { Bail }\end{array}$ & G & \\
\hline H-2-99732 & 1 & Drill String Burial Container & G & \\
\hline H-2-99733 & $1-2$ & $\begin{array}{l}\text { Drill String Burial Container } \\
\text { Assembly }\end{array}$ & G & \\
\hline H-2-99734 & 1 & $\begin{array}{l}\text { Drill String Burial Container } \\
\text { Detail }\end{array}$ & G & \\
\hline H-2-99735 & 1 & $\begin{array}{l}\text { Drill String Burial Container } \\
\text { Detail }\end{array}$ & G & \\
\hline Detail & $1-2$ & Cask Field Loading Stand & G & \\
\hline
\end{tabular}


HNF-2305

Rev. 1

Table 4.5 Core Sampling Support Equipment Drawings

\begin{tabular}{|l|c|l|c|l||}
\hline \multicolumn{1}{|c|}{ Dwg. } & Sht. & \multicolumn{1}{|c|}{ Description } & Cat. & Justification/Notes \\
\hline H-2-99739 & 1 & Handling Tools NCAW Cask & G & \\
\hline H-2-99740 & $1-2$ & Viewport Camera & G & \\
\hline H-2-99741 & 1 & $\begin{array}{l}\text { Drill String Burial Container } \\
\text { Support }\end{array}$ & G & \\
\hline H-2-99742 & 1 & Support Stand Detail & G & \\
\hline H-2-99743 & 1 & Support Stand Detail & G & \\
\hline H-2-99744 & 1 & Support Stand Detail & G & \\
\hline H-2-690045 & $1-4$ & Maintenance Platform & G & \\
\hline H-2-690124 & $1-4$ & Downforce Calibration Stand & G & \\
\hline H-2-690125 & $1-2$ & Grapple Hoist Test Stand & G & \\
\hline H-2-690126 & $1-4$ & Misc. Remote Handing Tools & G & \\
\hline H-2-690127 & 1 & Misc Equipment & G & \\
\hline H-2-690128 & $1-2$ & Riser Sleeve Assembly & G & \\
\hline H-2-690130 & $1-2$ & Int Drill Rod Removal Tool & G & \\
\hline H-2-690131 & $1-2$ & $\begin{array}{l}\text { Riser Sleeve Spray Wash } \\
\text { Assembly }\end{array}$ & G & \\
\hline H-2-690132 & 1 & Riser Adapter Assembly & G & \\
\hline H-2-690133 & $1-2$ & Sampler Changeout Assembly & G & \\
\hline H-2-690134 & $1-5$ & Drill String Arrangement & E & - Depicts containment \\
boundaries
\end{tabular}


HNF-2305

Rev. 1

Table 4.5 Core Sampling Support Equipment Drawings

\begin{tabular}{||c|c|l|c|l||}
\hline \multicolumn{1}{|c|}{ Dwg. } & Sht. & \multicolumn{1}{|c|}{ Description } & Cat. & Justification/Notes \\
\hline \hline H-2-690140 & $1-6$ & Core Sampler Assembly & S & $\begin{array}{l}\text { Note: Important for } \\
\text { Maintenance }\end{array}$ \\
\hline H-2-690141 & $1-2$ & Sampler Pull Test Tool & G & \\
\hline H-2-690144 & $1-4$ & Sampler Retrieval Tool Assy & G & \\
\hline H-2-821457 & $1-4$ & Drill String Arrangements & E & $\begin{array}{l}\text {-Depicts equipment } \\
\text { Essential to Safe Operation }\end{array}$ \\
\hline H-2-825312 & 1 & $\begin{array}{l}\text { Transfer Cask Modifications and } \\
\text { Lifting Bail }\end{array}$ & G & \\
\hline H-2-826513 & $1-2$ & Drill Rod Purge Installation & G & \\
\hline H-9-000174 & 1 & Purge Gas Trailer Assembly & G & $\begin{array}{l}\text { Information from this } \\
\text { drawings has been moved } \\
\text { to H-2-85299 }\end{array}$ \\
\hline H-14-021922 & $1-2$ & Breathing Air Compressor P\&ID & E & $\begin{array}{l}\text { - Process and } \\
\text { Instrumentation Diagram }\end{array}$ \\
\hline
\end{tabular}

\subsubsection{Summary for Core Sampling Support Equipment Drawings}

Table 4.5.1 Summary of Core Sampling Support Equipment Drawings

\begin{tabular}{||l|c|c|}
\hline \hline \multicolumn{1}{|c|}{ Category } & Drawings & Sheets \\
\hline General Drawings & 123 & 231 \\
\hline Support Drawings & 5 & 20 \\
\hline Essential Drawings & $7^{*}$ & 19 \\
\hline
\end{tabular}

"Note: Drawing H-2-85299 which has both general and Essential drawings sheets is included in the Essential Drawing total. 
HNF-2305

Rev. 1

\subsection{RMCST SAMPLING EXHAUSTER DRAWINGS}

Table 4.6 RMCST Exhauster Drawings

\begin{tabular}{|c|c|c|c|c|}
\hline Dwg. & Sht. & Description & Cat. & Justification/Notes \\
\hline H-14-100740 & $1-2$ & $\begin{array}{l}\text { Misc Detail RMCS Exhauster } \\
\text { C }\end{array}$ & $\mathrm{G}$ & \\
\hline H-14-100742 & $1-2$ & Breather Vent Stack & G & \\
\hline $\mathrm{H}-2-818186$ & $1-4$ & Exhauster Assembly & $\mathrm{G}$ & Used by AR Vault \\
\hline $\mathrm{H}-2-818188$ & 1 & Plenum Assembly & G & \\
\hline $\mathrm{H}-2-818189$ & $1-3$ & $\begin{array}{l}\text { RMCS External Skid } \\
\text { Electrical Details }\end{array}$ & $\mathrm{G}$ & Used by AR Vault \\
\hline $\mathrm{H}-2-818201$ & $1-2$ & $\begin{array}{l}\text { RMCS Skid Electrical } \\
\text { Assembly }\end{array}$ & $\mathrm{G}$ & \\
\hline $\mathrm{H}-2-818202$ & $1-4$ & Misc Detail Exhauster. & $\mathrm{G}$ & \\
\hline $\mathrm{H}-2-818204$ & 1 & Bottle Rack Assembly RMCS & $\mathrm{G}$ & Not used \\
\hline $\mathrm{H}-2-818205$ & 1 & $\begin{array}{l}\text { Pre Filter Stand Assembly } \\
\text { RMCS }\end{array}$ & $\mathrm{G}$ & Not used \\
\hline H-2-818206 & $1-2$ & Lift Assembly RMCS & $\mathrm{G}$ & \\
\hline $\mathrm{H}-2-829081$ & $1-2$ & Overall Assy Exhauster C & $S$ & \\
\hline $\mathrm{H}-2-829082$ & $1-6$ & $\begin{array}{l}\text { Mechanical Installation } \\
\text { Exhauster C }\end{array}$ & S & \\
\hline H-2-829083 & $1-3$ & $\begin{array}{l}\text { Stack Instrumentation } \\
\text { Exhauster C }\end{array}$ & S & \\
\hline $\mathrm{H}-2-829084$ & $1-3$ & $\begin{array}{l}\text { Sampling Exhauster Heater } \\
\text { Assembly Exhauster C }\end{array}$ & S & \\
\hline $\mathrm{H}-2-829085$ & $1-3$ & Skid Assembly Exhauster C & G & \\
\hline $\mathrm{H}-2-829086$ & $1-4$ & $\begin{array}{l}\text { Instrument \& Control Panel } \\
\text { Assembly Exhauster C }\end{array}$ & S & $\begin{array}{l}\text { Demonstrates Compliance } \\
\text { with Laws \& Regulations }\end{array}$ \\
\hline
\end{tabular}


HNF-2305

Rev. 1

Table 4.6 RMCST Exhauster Drawings

\begin{tabular}{|c|c|c|c|c|}
\hline Dwg. & Sht. & Description & Cat. & Justification/Notes \\
\hline $\mathrm{H}-2-829087$ & $1-5$ & $\begin{array}{l}\text { Stack Enclosure Assembly } \\
\text { Exhauster C }\end{array}$ & G & \\
\hline $\mathrm{H}-2-829088$ & $1-2$ & $\begin{array}{l}\text { Record Sampler Enclosure } \\
\text { Assembly Exhauster C }\end{array}$ & G & \\
\hline $\mathrm{H}-2-829089$ & $1-2$ & $\begin{array}{l}\text { Cam Probe Enclosure } \\
\text { Assembly Exhauster C }\end{array}$ & G & \\
\hline H-2-829090 & $1-2$ & $\begin{array}{l}\text { Lower Weather Shield } \\
\text { Assembly Exhauster C }\end{array}$ & G & \\
\hline H-2-829091 & $1-2$ & $\begin{array}{l}\text { Upper Weather Shield } \\
\text { Assembly Exhauster C }\end{array}$ & G & \\
\hline $\mathrm{H}-2-829100$ & 1 & Drawing Tree Exhauster C & $S$ & \\
\hline H-2-829105 & $1-5$ & Electrical Installation & S & \\
\hline $\mathrm{H}-2-829106$ & 1 & $\begin{array}{l}\text { Piping and Instrumentation } \\
\text { Diagram Exhauster C }\end{array}$ & $\mathrm{E}$ & \\
\hline $\mathrm{H}-2-829107$ & $1-2$ & $\begin{array}{l}\text { Variable Frequency Drive } \\
\text { Exhauster C }\end{array}$ & G & \\
\hline $\mathrm{H}-2-829108$ & $1-5$ & $\begin{array}{l}\text { Instrument Enclosure } \\
\text { Connection Diagram } \\
\text { Exhauster C }\end{array}$ & $\mathrm{S}$ & \\
\hline H-2-829109 & $1-7$ & $\begin{array}{l}\text { Exhauster Electrical } \\
\text { Elementary Dia. Exhauster c }\end{array}$ & S & \\
\hline $\mathrm{H}-2-829110$ & $1-3$ & $\begin{array}{l}\text { Exhauster Electrical } \\
\text { Interconnect Exhauster C }\end{array}$ & $\mathrm{E}$ & \\
\hline $\mathrm{H}-2-829111$ & $1-2$ & $\begin{array}{l}\text { Junction Box Detail } \\
\text { Exhauster C }\end{array}$ & G & \\
\hline $\mathrm{H}-2-829120$ & $1-2$ & Overall Assy Exhauster B & $\mathrm{S}$ & \\
\hline
\end{tabular}


HNF-2305

Rev. 1

Table 4.6 RMCST Exhauster Drawings

\begin{tabular}{|c|c|c|c|c|}
\hline Dwg. & Sht. & Description & Cat. & Justification/Notes \\
\hline $\mathrm{H}-2-829121$ & $1-6$ & $\begin{array}{l}\text { Mechanical Installation } \\
\text { Exhauster B }\end{array}$ & $S$ & \\
\hline H-2-829122 & $1-3$ & $\begin{array}{l}\text { Stack Instrumentation } \\
\text { Exhauster B }\end{array}$ & S & \\
\hline H-2-829123 & $1-3$ & $\begin{array}{l}\text { Sampling Exhauster Heater } \\
\text { Assembly Exhauster B }\end{array}$ & $S$ & \\
\hline H-2-829124 & $1-3$ & Skid Assembly Exhauster B & G & \\
\hline H-2-829125 & $1-4$ & $\begin{array}{l}\text { Instrument \& Control Panel } \\
\text { Assembly Exhauster B }\end{array}$ & S & \\
\hline H-2-829126 & $1-5$ & $\begin{array}{l}\text { Stack Enclosure Assembly } \\
\text { Exhauster B }\end{array}$ & G & \\
\hline H-2-829127 & $1-2$ & $\begin{array}{l}\text { Record Sampler Enclosure } \\
\text { Assembly Exhauster B }\end{array}$ & G & \\
\hline H-2-829128 & $1-2$ & $\begin{array}{l}\text { Cam Probe Enclosure } \\
\text { Assembly Exhauster B }\end{array}$ & G & \\
\hline H-2-829129 & $1-2$ & $\begin{array}{l}\text { Lower Weather Shield } \\
\text { Assembly Exhauster B }\end{array}$ & G & \\
\hline H-2-829130 & $1-2$ & $\begin{array}{l}\text { Upper Weather Shield } \\
\text { Assembly Exhauster B }\end{array}$ & G & \\
\hline H-2-829134 & 1 & $\begin{array}{l}\text { Piping and Instrumentation } \\
\text { Diagram Exhauster B }\end{array}$ & $\mathrm{E}$ & \\
\hline H-2-829135 & $1-5$ & $\begin{array}{l}\text { Electrical Installation } \\
\text { Exhauster B }\end{array}$ & S & . \\
\hline H-2-829.136 & $1-2$ & $\begin{array}{l}\text { Variable Frequency Drive } \\
\text { Exhauster B }\end{array}$ & G & \\
\hline H-2-829137 & $1-5$ & $\begin{array}{l}\text { Instrument Enclosure } \\
\text { Connection Diagram } \\
\text { Exhauster B }\end{array}$ & $\mathrm{S}$ & \\
\hline
\end{tabular}


HNF-2305

Rev. 1

Table 4.6 RMCST Exhauster Drawings

\begin{tabular}{||c|c|l|c|l||}
\hline \hline Dwg. & Sht. & \multicolumn{1}{c|}{ Description } & Cat. & Justification/Notes \\
\hline \hline H-2-829138 & $1-7$ & $\begin{array}{l}\text { Exhauster Electrical } \\
\text { Elementary Dia. Exhauster C }\end{array}$ & S & \\
\hline H-2-829139 & $1-3$ & $\begin{array}{l}\text { Exhauster Electrical } \\
\text { Interconnect Exhauster B }\end{array}$ & E & \\
\hline H-2-829140 & 1 & $\begin{array}{l}\text { Junction Box Detail } \\
\text { Exhauster B }\end{array}$ & G & \\
\hline H-2-829141 & 1 & Drawing Tree Exhauster B & S & Important to Maintenance \\
\hline
\end{tabular}

\subsubsection{Summary for RMCST Exhauster Drawings}

Table 4.6.1 Summary for RMCST Exhauster Drawings

\begin{tabular}{|l|c|c|}
\hline \multicolumn{1}{|c|}{ Category } & Drawings & Sheets \\
\hline General Drawings & 26 & 60 \\
\hline Support Drawings. & 18 & 72 \\
\hline Essential Drawings. & 4 & 8 \\
\hline
\end{tabular}

\subsection{OTHER SAMPLING EQUIPMENT DRAWINGS}

Table 4.7 Other Sampling Equipment Drawings

\begin{tabular}{|c|c|l|c|c||}
\hline Dwg. & Sht. & Description & Cat. & Justification/Notes \\
\hline \hline & & AUGER SAMPLING DRAWINGS & & \\
\hline H-2-79960 & $1-7$ & Surface Sampler Auger Assembly & G & \\
\hline H-2-826321 & $1-2$ & Auger Bit & G & \\
\hline
\end{tabular}


HNF-2305

Rev. 1

Table 4.7 Other Sampling Equipment Drawings

\begin{tabular}{|c|c|l|c|l||}
\hline \multicolumn{1}{|c|}{ Dwg. } & Sht. & \multicolumn{1}{|c|}{ Description } & Cat. & Justification/Notes \\
\hline \hline & & GRAB SAMPLING DRAWINGS & & \\
\hline H-2-37844 & $1-3$ & $\begin{array}{l}\text { Instrumentation Miscellaneous } \\
\text { Installation Details }\end{array}$ & S & \\
\hline H-2-85602 & $1-5$ & Supernatant and Sludge Sampler & S & \\
\hline H-2-85645 & $1-2$ & $\begin{array}{l}\text { Tri-Pod and Bottle Hoist for } \\
\text { Supernatant and Sludge Sampler }\end{array}$ & S & \\
\hline H-2-826597 & $1-2$ & $\begin{array}{l}\text { Table Assembly for Supernatant } \\
\text { and Sludge Sampler }\end{array}$ & S & \\
\hline & & VAPOR SAMPLING DRAWINGS & & \\
\hline H-2-815402 & $1-2$ & $\begin{array}{l}\text { VSS Piping and Instrumentation } \\
\text { Diagram }\end{array}$ & G & Not Used \\
\hline H-2-815403 & 1 & VSS Mimic Panel Assemblies & G & Not Used \\
\hline H-2-815404 & $1-2$ & VSS Control Rack Assemblies & G & Not Used \\
\hline H-2-815405 & $1-2$ & VSS I/O Rack Module Assy & G & Not Used \\
\hline H-2-815406 & 1 & VSS Drawing Index Tree & G & Not Used \\
\hline H-2-815407 & $1-3$ & $\begin{array}{l}\text { VSS Exterior Component Rack } \\
\text { Assemblies }\end{array}$ & G & Not Used \\
\hline H-2-815408 & $1-19$ & VSS Control Diagram & G & Not Used \\
\hline H-2-815409 & $1-3$ & $\begin{array}{l}\text { VSS Control Rack Interconnect } \\
\text { Diagram }\end{array}$ & G & Not Used \\
\hline H-2-815410 & $1-2$ & $\begin{array}{l}\text { VSS I/O Rack Interconnect } \\
\text { Diagrams }\end{array}$ & G & Not Used \\
\hline H-2-815411 & 1 & VSS Cabling Arrangement & G & Not Used \\
\hline H-2-815412 & $1-3$ & $\begin{array}{l}\text { VSS Interior Interconnect } \\
\text { Diagram }\end{array}$ & G & Not Used \\
\hline & & & & \\
\hline & & & & \\
\hline & & & \\
\hline
\end{tabular}


HNF-2305

Rev. 1

Table 4.7 Other Sampling Equipment Drawings

\begin{tabular}{||c|c|l|c|l||}
\hline Dwg. & Sht. & \multicolumn{1}{|c|}{ Description } & Cat. & \multicolumn{1}{|c|}{ Justification/Notes } \\
\hline \hline H-2-815413 & 1 & VSS Solid State Relay Panel Assy & G & Not Used \\
\hline H-2-815414 & $1-5$ & VSS Facility Interior Electrical & G & Not Used \\
\hline H-2-815415 & 1 & VSS Cable Assemblies & G & Not Used \\
\hline H-2-815417 & 1 & $\begin{array}{l}\text { VSS Intrinsic Barrier Box } \\
\text { Assembly }\end{array}$ & G & Not Used \\
\hline H-2-815418 & 1 & $\begin{array}{l}\text { VSS Heat Control Elementary } \\
\text { Diagram }\end{array}$ & G & Not Used \\
\hline H-2-815419 & $1-6$ & VSS Mechanical Installation & G & Not Used \\
\hline & & \multicolumn{1}{|l|}{ TYPE 4 } & & \\
\hline H-2-825301 & 1 & $\begin{array}{l}\text { In-Situ Sample Head Assembly \& } \\
\text { Details }\end{array}$ & $\mathrm{S}$ & \\
\hline H-2-825313 & $1-7$ & $\begin{array}{l}\text { Vapor Sampling Cart Assembly \& } \\
\text { Details }\end{array}$ & $\mathrm{S}$ & \\
\hline H-2-825314 & $1-2$ & $\begin{array}{l}\text { Vapor Sampling Cart Electrical } \\
\text { Installation and Interconnect } \\
\text { Diagram }\end{array}$ & $\mathrm{S}$ & \\
\hline
\end{tabular}

\subsubsection{Summary for Other Sampling Equipment Drawings}

Table 4.7.1 Summary for Other Sampling Equipment Drawings

\begin{tabular}{||l|c|c||}
\hline \multicolumn{1}{|c|}{ Category } & Drawings & Sheets \\
\hline General Drawings & 19 & 63 \\
\hline Support Drawings. & 7 & 22 \\
\hline Essential Drawings. & 0 & 0 \\
\hline
\end{tabular}


HNF-2305

Rev. 1

\subsection{LIGHT DUTY UTILITY ARM (LDUA) DRAWINGS}

Table 4.8 Light Duty Utility Arm (LDUA) Drawings

\begin{tabular}{||c|c|l|c|l||}
\hline \multicolumn{1}{|c|}{ Dwg. } & Sht. & \multicolumn{1}{|c|}{ Description } & Cat. & \multicolumn{1}{|c||}{ Justification/Notes } \\
\hline \hline H-6-14055 & 1 & LDUA System Block Dia & G & Not Deployed \\
\hline H-6-14057 & $1-2$ & LDUA Control System Block Dia & G & Not Deployed \\
\hline H-6-14099 & 1 & LDUA Purge Air Supply Assy & G & Not Deployed \\
\hline H-6-14100 & $1-7$ & $\begin{array}{l}\text { LDUA Operations Control Trailer } \\
\text { Hanford }\end{array}$ & G & Not Deployed \\
\hline H-6-14101 & 1 & $\begin{array}{l}\text { Wiring Diagram, Air Conditioning } \\
\text { Control \& Power, Hanford }\end{array}$ & G & Not Deployed \\
\hline H-6-14102 & 1 & $\begin{array}{l}\text { Electrical Layout, Service } \\
\text { Equipment \& Grounding Scheme } \\
\text { Hanford }\end{array}$ & G & Not Deployed \\
\hline H-6-14103 & 1 & Diagram, HVAC Piping Hanford & G & Not Deployed \\
\hline H-6-14104 & 1 & $\begin{array}{l}\text { LDUA - OCT Ventilation Control } \\
\text { Panel }\end{array}$ & G & Not Deployed \\
\hline H-6-14105 & 1 & $\begin{array}{l}\text { Cable, Trailer Power Supply, } \\
\text { 480V/3PH }\end{array}$ & G & Not Deployed \\
\hline H-6-14106 & $1-2$ & $\begin{array}{l}\text { Operation Control Trailer } \\
\text { Instrument Rack Equipment } \\
\text { Arrangement }\end{array}$ & G & Not Deployed \\
\hline H-6-14107 & 1 & $\begin{array}{l}\text { LDUA Operations Control Trailer } \\
\text { LAN and Video Cable Layout }\end{array}$ & G & Not Deployed \\
\hline H-6-14109 & 1 & $\begin{array}{l}\text { LDUA System Power Distribution } \\
\text { One-Line Dia. }\end{array}$ & G & Not Deployed \\
\hline H-6-14110 & $1-3$ & $\begin{array}{l}\text { LDUA Power Distribution Rack } \\
\text { LDUA Power Distribution Skid, } \\
\text { etc. }\end{array}$ & G & Not Deployed \\
\hline Not Deployed \\
\hline
\end{tabular}


HNF-2305

Rev. 1

Table 4.8 Light Duty Utility Arm (LDUA) Drawings

\begin{tabular}{||c|c|l|c|l||}
\hline \multicolumn{1}{|c|}{ Dwg. } & Sht. & \multicolumn{1}{|c|}{ Description } & Cat. & \multicolumn{1}{|c|}{ Justification/Notes } \\
\hline H-6-14112 & $1-2$ & $\begin{array}{l}\text { LDUA System Power Distribution } \\
\text { Cable Assys }\end{array}$ & G & Not Deployed \\
\hline H-6-14113 & 1 & $\begin{array}{l}\text { LDUA Fiber Optic Umbilical } \\
\text { Interconnection Dia. }\end{array}$ & G & Not Deployed \\
\hline H-6-14114 & 1 & $\begin{array}{l}\text { Control Network Interconnection } \\
\text { Dia. }\end{array}$ & G & Not Deployed \\
\hline H-6-14115 & 1 & $\begin{array}{l}\text { I/O Subsystem Interconnection } \\
\text { Dia. }\end{array}$ & G & Not Deployed \\
\hline H-6-14116 & 1 & $\begin{array}{l}\text { I/O Subsystem Converter and } \\
\text { Terminal Block Assy }\end{array}$ & G & Not Deployed \\
\hline H-6-14117 & $1-2$ & $\begin{array}{l}\text { Connector Adapter Module } \\
\text { Connection Dia. Etc. }\end{array}$ & G & Not Deployed \\
\hline H-6-14118 & 1 & $\begin{array}{l}\text { Video Display and Recording Sys } \\
\text { Interconnection Dia }\end{array}$ & G & Not Deployed \\
\hline H-6-14119 & $1-2$ & $\begin{array}{l}\text { Video System Panels General } \\
\text { Arrangement }\end{array}$ & G & Not Deployed \\
\hline H-6-14120 & 1 & $\begin{array}{l}\text { Video Display and Recording } \\
\text { System Cable Assy }\end{array}$ & G & Not Deployed \\
\hline H-6-14121 & 1 & I/O Subsystem Cable Assy & G & Not Deployed \\
\hline H-6-14125 & $1-4$ & ATIE Assy & G & Not Deployed \\
\hline H-6-14126 & $1-2$ & $\begin{array}{l}\text { ATIE Elementary Diagram and } \\
\text { Panelboard Schedule }\end{array}$ & G & Not Deployed \\
\hline H-6-14127 & 1 & ATIE EE Patch Panel, 4300A2A1 & G & Not Deployed \\
\hline H-6-14128 & 1 & $\begin{array}{l}\text { ATIE OPTO-22 Drawer \#1, } \\
\text { 4220A2 }\end{array}$ & G & Not Deployed \\
\hline H-6-14129 & 1 & $\begin{array}{l}\text { ATIE Interlock Junction Drawer } \\
\text { 4300A1A2 }\end{array}$ & G & Not Deployed \\
\hline
\end{tabular}


HNF-2305

Rev. 1

Table 4.8 Light Duty Utility Arm (LDUA) Drawings

\begin{tabular}{||c|c|l|c|l||}
\hline \multicolumn{1}{|c|}{ Dwg. } & Sht. & \multicolumn{1}{|c|}{ Description } & Cat. & \multicolumn{1}{|c|}{ Justification/Notes } \\
\hline H-6-14130 & 1 & $\begin{array}{l}\text { At-Tank Instr. Enclosure } \\
\text { Equipment Arrangement }\end{array}$ & G & Not Deployed \\
\hline H-6-14131 & $1-2$ & $\begin{array}{l}\text { At-Tank Instr. Encl. ATIE } \\
\text { Interconnection Dia.. }\end{array}$ & G & Not Deployed \\
\hline H-6-14133 & 1 & $\begin{array}{l}\text { VPTO-22 Drawer No. 2 Wiring } \\
\text { Dia. }\end{array}$ & G & Not Deployed \\
\hline H-6-14134 & 1 & $\begin{array}{l}\text { ATIE Interlock Junction Drawer } \\
\text { Wiring Dia. }\end{array}$ & G & Not Deployed \\
\hline H-6-14135 & $1-3$ & $\begin{array}{l}\text { At Tank Instr Enclosure ATIE } \\
\text { Internal Cable Assy }\end{array}$ & G & Not Deployed \\
\hline H-6-14140 & $1-3$ & LDUA Laser Alignment Details & G & Not Deployed \\
\hline H-6-14142 & $1-2$ & $\begin{array}{l}\text { Operation Overview Video } \\
\text { Camera System }\end{array}$ & G & Not Deployed \\
\hline H-6-14145 & $1-2$ & LDUA System Misc Cable Assy & G & Not Deployed \\
\hline H-6-14150 & $1-2$ & Pneumatic System P \& ID & G & Not Deployed \\
\hline H-6-14152 & 1 & $\begin{array}{l}\text { Modified LDUA Outer Tube and } \\
\text { Sheave Support Assy }\end{array}$ & G & Not Deployed \\
\hline H-6-14160 & 1 & Tooling Interface Plate Assy & G & Not Deployed \\
\hline H-6-14170 & 1 & $\begin{array}{l}\text { LDUA Subsystem Controller } \\
\text { Pendant Arrangement }\end{array}$ & G & Not Deployed \\
\hline H-6-14171 & 1 & $\begin{array}{l}\text { LDUA Console Subsystem } \\
\text { Control Panel Arrangement }\end{array}$ & G & Not Deployed \\
\hline H-6-14172 & $1-3$ & Power Interface Module (LDUA) & G & Not Deployed \\
\hline H-6-14173 & 1 & Boot Clamp Elbow, Wrist Assy & G & Not Deployed \\
\hline H-6-14174 & $1-9$ & LDUA System Name plate Sched. & G & Not Deployed \\
\hline
\end{tabular}


HNF-2305

Rev. 1

Table 4.8 Light Duty Utility Arm (LDUA) Drawings

\begin{tabular}{|c|c|c|c|c|}
\hline Dwg. & Sht. & Description & Cat. & Justification/Notes \\
\hline H-6-14175 & $1-2$ & $\begin{array}{l}\text { LDUA Truck Power Purge } \\
\text { Elementary }\end{array}$ & G & Not Deployed \\
\hline H-6-14176 & $1-2$ & LDUA In-Tank Materials & G & Not Deployed \\
\hline H-6-14177 & $1-2$ & $\begin{array}{l}\text { LDUA Outrigger Hydraulic } \\
\text { Schematic }\end{array}$ & G & Not Deployed \\
\hline H-6-14180 & $1-2$ & $\begin{array}{l}\text { Brake Platform Mobile } \\
\text { Deployment System }\end{array}$ & G & Not Deployed \\
\hline H-6-14185 & 1 & $\begin{array}{l}\text { LDUA Shoulder Cable Harness } \\
\text { Wiring Diagram }\end{array}$ & G & Not Deployed \\
\hline H-6-14186 & $1-4$ & $\begin{array}{l}\text { LDUA Subsystem Controller } \\
\text { Wiring Diagram }\end{array}$ & G & Not Deployed \\
\hline$H-6-14200$ & 1 & $\begin{array}{l}\text { UST-ID Overview Video Camera } \\
\text { System, OVS, Control Box } \\
\text { Schematic }\end{array}$ & G & Not Deployed \\
\hline H-6-14201 & 1 & $\begin{array}{l}\text { UST-ID OVS Cable Reel } \\
\text { Schematic }\end{array}$ & G & Not Deployed \\
\hline H-6-14202 & 1 & $\begin{array}{l}\text { UST-ID OVS Camera Housting } \\
\text { Schematic }\end{array}$ & G & Not Deployed \\
\hline $\mathrm{H}-6-14203$ & 1 & $\begin{array}{l}\text { UST-ID Video Systems User End } \\
\text { Schematic }\end{array}$ & G & Not Deployed \\
\hline H-6-14204 & 1 & $\begin{array}{l}\text { UST-ID OSVS Camera Housing } \\
\text { Schematic }\end{array}$ & G & Not Deployed \\
\hline H-6-14205 & $1-3$ & $\begin{array}{l}\text { Overview Video System OVS } \\
\text { Details }\end{array}$ & G & Not Deployed \\
\hline H-6-14206 & 1 & $\begin{array}{l}\text { Overview Video System OVS } \\
\text { Lamp Housing Weldment }\end{array}$ & G & Not Deployed \\
\hline H-6-14207 & $1-3$ & $\begin{array}{l}\text { OVS and OSVS Video Systems } \\
\text { Overview Details }\end{array}$ & G & Not Deployed \\
\hline
\end{tabular}


HNF-2305

Rev. 1

Table 4.8 Light Duty Utility Arm (LDUA) Drawings

\begin{tabular}{||c|c|l|c|l||}
\hline Dwg. & Sht. & \multicolumn{1}{|c|}{ Description } & Cat. & \multicolumn{1}{|c||}{ Justification/Notes } \\
\hline \hline H-6-14208 & 1 & $\begin{array}{l}\text { OVS and OSVS Video Systems } \\
\text { Overview Housing Weldments }\end{array}$ & G & Not Deployed \\
\hline H-6-14209 & $1-4$ & $\begin{array}{l}\text { OVS and OSVS Video System } \\
\text { Deployment Stand Assy }\end{array}$ & G & Not Deployed \\
\hline H-6-14210 & 1 & $\begin{array}{l}\text { OVS and OSVS Video System } \\
\text { Deployment Stand Assy -Det. }\end{array}$ & G & Not Deployed \\
\hline H-6-14211 & 1 & $\begin{array}{l}\text { OVS And OSVS Video Systems } \\
\text { Deployment Stand Frame Subassy }\end{array}$ & G & Not Deployed \\
\hline H-6-14212 & 1 & $\begin{array}{l}\text { OVS and OSVS Video Systems } \\
\text { Deployment Stand Details-Ribbon } \\
\text { Cable Drum }\end{array}$ & G & Not Deployed \\
\hline H-6-14213 & 1 & $\begin{array}{l}\text { OVS and OSVS Video Systems } \\
\text { Deployment Stand Details - } \\
\text { Regular Cable Drum }\end{array}$ & G & Not Deployed \\
\hline H-6-14214 & 1 & $\begin{array}{l}\text { OVS and OSVS Video Systems } \\
\text { Deployment Stand Details-Box } \\
\text { Subassembly }\end{array}$ & G & Not Deployed \\
\hline H-6-14217 & 1 & $\begin{array}{l}\text { OVS and OSVS Video Systgems } \\
\text { Deployment Stand Details-Camera } \\
\text { HSG Sleeve }\end{array}$ & G & Not Deployed \\
\hline H-6-14218 & 1 & $\begin{array}{l}\text { VICON Transmitter PC Board } \\
\text { Interface }\end{array}$ & G & Not Deployed \\
\hline H-6-14242 & $1-2$ & $\begin{array}{l}\text { High Resolution Stereoscopic } \\
\text { Video Camera Sys HRSVS }\end{array}$ & G & Not Deployed \\
\hline H-6-14243 & $1-3$ & $\begin{array}{l}\text { High Resolution Stereoscopic } \\
\text { Video Camera Sys HRSVS }\end{array}$ & G & Not Deployed \\
\hline & 14216 & $\begin{array}{l}\text { OVS and OSV Systems } \\
\text { Deplans Misc. Details }\end{array}$ & G & Not Deployed \\
\hline
\end{tabular}


HNF-2305

Rev. 1

Table 4.8 Light Duty Utility Arm (LDUA) Drawings

\begin{tabular}{||c|c|l|c|l||}
\hline \multicolumn{1}{|c|}{ Dwg. } & Sht. & \multicolumn{1}{|c|}{ Description } & Cat. & \multicolumn{1}{|c||}{ Justification/Notes } \\
\hline H-6-14244 & 1 & $\begin{array}{l}\text { High Resolution Stereoscopic } \\
\text { Video Camera Sys HRSVS }\end{array}$ & G & Not Deployed \\
\hline H-6-14245 & 1 & $\begin{array}{l}\text { High Resolution Stereoscopic } \\
\text { Video Camera Sys HRSVS }\end{array}$ & G & Not Deployed \\
\hline H-6-14246 & 1 & $\begin{array}{l}\text { Closeup Stereo Video System } \\
\text { Camera Housing Schematic }\end{array}$ & G & Not Deployed \\
\hline H-6-14270 & $1-8$ & $\begin{array}{l}\text { CVEE and HRSVS End to End } \\
\text { Dwg-Det }\end{array}$ & G & Not Deployed \\
\hline H-6-14271 & $1-3$ & WHC Power Chassis & G & Not Deployed \\
\hline H-6-14279 & 1 & $\begin{array}{l}\text { Common Video End Effector } \\
\text { CVEE Support Electronics }\end{array}$ & G & Not Deployed \\
\hline H-6-14296 & 1 & $\begin{array}{l}\text { End Effector Handling System } \\
\text { Weight Assy }\end{array}$ & G & Not Deployed \\
\hline H-6-14297 & $1-2$ & LDUA Riser Isolation Valve Adap & G & Not Deployed \\
\hline H-6-14298 & 1 & $\begin{array}{l}\text { LDUA TRIC Storage and } \\
\text { Transport Stand }\end{array}$ & G & Not Deployed \\
\hline H-6-14299 & 1 & LDUA TRIC Rigging Fixture & G & Not Deployed \\
\hline H-6-14301 & $1-3$ & $\begin{array}{l}\text { Enclosure, TRIC, Specification } \\
\text { Control Diagram }\end{array}$ & G & Not Deployed \\
\hline H-6-14302 & 1 & Plate, Interface TRIC Enclosure & G & Not Deployed \\
\hline H-6-14303 & 1 & Angle Mounting TRIC Enclosure & G & Not Deployed \\
\hline H-6-14304 & $1-8$ & TRIC, Enclosure Assembly & G & Not Deployed \\
\hline H-6-14305 & $1-8$ & End Effector Handling Sys Rails & G & Not Deployed \\
\hline H-6-14306 & $1-4$ & Cart Assembly & G & Not Deployed \\
\hline H-6-14307 & $1-9$ & End Effector Transport Bar & G & Not Deployed \\
\hline H-6-14308 & 1 & VPM Adapter & G & Not Deployed \\
\hline
\end{tabular}


HNF-2305

Rev. 1

Table 4.8 Light Duty Utility Arm (LDUA) Drawings

\begin{tabular}{|c|c|c|c|c|}
\hline Dwg. & Sht. & Description & Cat. & Justification/Notes \\
\hline H-6-14320 & $1-7$ & Gripper End Effector Assy & G & Not Deployed \\
\hline H-6-14321 & $1-8$ & Camera Housing Assemblies & $\mathrm{G}$ & Not Deployed \\
\hline H-6-14322 & $1-5$ & Gripper Assy & $\mathrm{G}$ & Not Deployed \\
\hline $\mathrm{H}-6-14323$ & 1 & GEE Electrical Schematic & G & Not Deployed \\
\hline $\mathrm{H}-6-14324$ & 1 & Cable Assy & $\mathrm{G}$ & Not Deployed \\
\hline H-6-14325 & 1 & Pneumatic Block Diagram & G & Not Deployed \\
\hline H-6-14326 & $1-2$ & Pneumatic Rack Mount Assy & $\mathrm{G}$ & Not Deployed \\
\hline H-6-14340 & $1-13$ & Decon Module Assy. & G & Not Deployed \\
\hline H-6-14341 & 1 & $\begin{array}{l}\text { LDUA Water Decon Trailer } \\
\text { Piping Schematic Diagram }\end{array}$ & G & Not Deployed \\
\hline $\mathrm{H}-6-14342$ & $1-2$ & $\begin{array}{l}\text { Water Decon Power and Control } \\
\text { Wiring Diagram }\end{array}$ & $\mathrm{G}$ & Not Deployed \\
\hline $\mathrm{H}-6-14345$ & $1-4$ & Mounting Platform Assy & $\mathrm{G}$ & Not Deployed \\
\hline H-6-15012 & 1 & $\begin{array}{l}\text { EREE Controller Electrical } \\
\text { Schematic }\end{array}$ & $\mathrm{G}$ & Not Deployed \\
\hline $\mathrm{H}-6-15013$ & 1 & EREE Electrical Schematic & $\mathrm{G}$ & Not Deployed \\
\hline
\end{tabular}


HNF-2305

Revil 1

\subsubsection{Summary for Light Duty Utility Arm Drawings}

Table 4.8.1 Summary for Other Sampling Equipment Drawings

\begin{tabular}{||l|c|c|}
\hline \multicolumn{1}{|c|}{ Category } & Drawings & Sheets \\
\hline General Drawings & 103 & 227 \\
\hline Support Drawings. & 0 & 0 \\
\hline Essential Drawings. & 0 & 0 \\
\hline
\end{tabular}


HNF-2305

Rev. 1

\subsection{SUMMARY}

The results of the drawing evaluation of Characterization sampling equipment are summarized in Table 5.1. The table shows both number of drawings and number of sheets.

Table 5.1 Drawing Evaluation Summary

\begin{tabular}{|c|c|c|c|}
\hline \multirow{2}{*}{ Equipment } & \multicolumn{3}{|c|}{ Drawing Category } \\
\hline & $\begin{array}{c}\text { General } \\
\text { (Dwgs/Sheets) }\end{array}$ & $\begin{array}{c}\text { Support } \\
\text { (Dwgs/Sheets) }\end{array}$ & $\begin{array}{c}\text { Essential } \\
\text { (Dwgs/Sheets) }\end{array}$ \\
\hline Truck 1 & $28 / 40$ & $13 / 44$ & $5 / 14$ \\
\hline Truck $1 \& 2$ & $28 / 46$ & $2 / 4$ & $0 / 0$ \\
\hline Truck 2 & $60 / 212$ & $0 / 0$ & $0 / 0$ \\
\hline Truck $3 \& 4$ & $59 / 138$ & $18 / 94$ & $8 / 50$ \\
\hline Support Equipment & $123 / 231$ & $4 / 19$ & $7 / 19$ \\
\hline Exhauster & $26 / 60$ & $18 / 72$ & $4 / 8$ \\
\hline $\begin{array}{l}\text { Other Sampling } \\
\text { Equipment }\end{array}$ & $19 / 63$ & $7 / 22$ & $0 / 0$ \\
\hline LDUA & $103 / 227$ & $0 / 0$ & $0 / 0$ \\
\hline TOTALS & $446 / 1015$ & $62 / 255$ & 24/91 \\
\hline \multicolumn{2}{|c|}{$\begin{array}{l}\text { GRAND TOTAL of Characterization } \\
\text { Drawings (Dwgs/Sheets) }\end{array}$} & \multicolumn{2}{|c|}{$527 / 1355$} \\
\hline
\end{tabular}

Currently, all Truck $3 \& 4$, exhauster, and support equipment essential/support drawings have been established and are being maintained. The Characterization Equipment Essential/Support Drawing Plan (HNF, 1999b) is also being maintained to document the sampling equipment drawing category. Truck 2 and LDUA drawings have been assigned a general drawing category since Truck 2 is on standby and LDUA is not deployed. Truck 1 essential/support drawings have not been established due to uncertainty about future use. 


\section{HNF -2305}

Rev. 1

\subsection{REFERENCES}

Wilson, 1997, HNF-SD-WM-ETP-216, Rev. 1, Engineering Task Plan for Truck 3 \& 4 Drawing Compliance and Evaluation, Lockheed Martin Hanford Corporation, Richland, Washington

HNF, 1999a, HNF-IP-0842, Sec 4.25, TWRS Administation, Engineering Drawings, Lockheed Martin Hanford Corporation, Richland, Washington

HNF, 1999b, HNF-3240, Rev. 3, Characterization Equipment Essential/Support Drawing Plan, Lockheed Martin Hanford Corporation, Richland, Washington. 